\title{
Stellar granulation as seen in disk-integrated intensity
}

\section{Theoretical scaling relations compared with observations ${ }^{\star}$}

\author{
R. Samadi ${ }^{1}$, K. Belkacem ${ }^{1}$, H.-G. Ludwig ${ }^{2,3}$, E. Caffau ${ }^{2,3}$, T. L. Campante ${ }^{4}$, G. R. Davies ${ }^{4}$, T. Kallinger ${ }^{5,6}$,

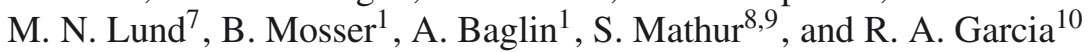 \\ ${ }^{1}$ LESIA, Observatoire de Paris, CNRS UMR 8109, UPMC, Université Denis Diderot, 5 place Jules Janssen, 92195 Meudon Cedex, \\ France \\ e-mail: reza.samadi@obspm.fr \\ 2 Zentrum für Astronomie der Universität Heidelberg, Landessternwarte, Königstuhl 12, 69117 Heidelberg, Germany \\ 3 GEPI, Observatoire de Paris, CNRS UMR 8111, Université Denis Diderot, 5 place Jules Janssen, 92195 Meudon Cedex, France \\ 4 School of Physics and Astronomy, University of Birmingham, Edgbaston, Birmingham, B15 2TT, UK \\ 5 Institute for Astronomy (IfA), University of Vienna, Türkenschanzstrasse 17, 1180 Vienna, Austria \\ ${ }^{6}$ Instituut voor Sterrenkunde, K.U. Leuven, Celestijnenlaan 200D, 3001 Leuven, Belgium \\ 7 Stellar Astrophysics Centre, Department of Physics and Astronomy, Aarhus University, Ny Munkegade 120, 8000 Aarhus C, \\ Denmark \\ 8 High Altitude Observatory, NCAR, PO Box 3000, Boulder, CO 80307, USA \\ 9 Space Science Institute, 4750 Walnut street Suite 205, Boulder, CO 80301, USA \\ 10 Laboratoire AIM, CEA/DSM CNRS Université Paris Diderot IRFU/SAp, 91191 Gif-sur-Yvette Cedex, France
}

Received 29 November 2012 / Accepted 26 July 2013

\begin{abstract}
Context. A large set of stars observed by CoRoT and Kepler shows clear evidence for the presence of a stellar background, which is interpreted to arise from surface convection, i.e., granulation. These observations show that the characteristic time-scale $\left(\tau_{\text {eff }}\right)$ and the root-mean-square (rms) brightness fluctuations $(\sigma)$ associated with the granulation scale as a function of the peak frequency $\left(v_{\max }\right)$ of the solar-like oscillations.

Aims. We aim at providing a theoretical background to the observed scaling relations based on a model developed in Paper I.

Methods. We computed for each 3D model the theoretical power density spectrum (PDS) associated with the granulation as seen in disk-integrated intensity on the basis of the theoretical model published in Paper I. For each PDS we derived the associated characteristic time $\left(\tau_{\text {eff }}\right)$ and the rms brightness fluctuations $(\sigma)$ and compared these theoretical values with the theoretical scaling relations derived from the theoretical model and the measurements made on a large set of Kepler targets.

Results. We derive theoretical scaling relations for $\tau_{\text {eff }}$ and $\sigma$, which show the same dependence on $v_{\max }$ as the observed scaling relations. In addition, we show that these quantities also scale as a function of the turbulent Mach number $\left(\mathcal{M}_{\mathrm{a}}\right)$ estimated at the photosphere. The theoretical scaling relations for $\tau_{\text {eff }}$ and $\sigma$ match the observations well on a global scale. Quantitatively, the remaining discrepancies with the observations are found to be much smaller than previous theoretical calculations made for red giants.

Conclusions. Our modelling provides additional theoretical support for the observed variations of $\sigma$ and $\tau_{\text {eff }}$ with $v_{\max }$. It also highlights the important role of $\mathcal{M}_{\mathrm{a}}$ in controlling the properties of the stellar granulation. However, the observations made with Kepler on a wide variety of stars cannot confirm the dependence of our scaling relations on $\mathcal{M}_{\mathrm{a}}$. Measurements of the granulation background and detections of solar-like oscillations in a statistically sufficient number of cool dwarf stars will be required for confirming the dependence of the theoretical scaling relations with $\mathcal{M}_{\mathrm{a}}$.
\end{abstract}

Key words. convection - turbulence - Sun: granulation - stars: oscillations - stars: atmospheres

\section{Introduction}

Since the launch of CoRoT (December 2006) and Kepler (March 2009), it is possible to accurately characterise the properties of the stellar granulation in other stars than the Sun (Michel et al. 2008; Ludwig et al. 2009a; Kallinger \& Matthews 2010; Chaplin et al. 2011b; Mathur et al. 2011). A very large number of stars has been observed on a long term by CoRoT and Kepler. A large set of them clearly show both solar-like oscillations and a stellar background signal, which is interpreted to be due to granulation at the surface of these stars. These observations show

\footnotetext{
^ Appendices are available in electronic form at http://www . aanda.org
}

that the characteristic time-scale of the granulation scales as the inverse of the peak frequency $\left(v_{\max }\right)$ of the solar-like oscillation spectra detected in these stars (Kallinger \& Matthews 2010; Mathur et al. 2011). In turn, the peak frequency $v_{\max }$ is known to scale as the cutoff-frequency $v_{\mathrm{c}}$ of the atmosphere (Brown et al. 1991; Kjeldsen \& Bedding 1995; Stello et al. 2009; Huber et al. 2009; Mosser et al. 2010; Belkacem et al. 2011). The observations of the stellar granulation background also reveal that the rms brightness fluctuations $(\sigma)$ associated with the granulation scales approximately as $v_{\max }^{-1 / 2}$ (Mathur et al. 2011; Chaplin et al. 2011b).

The measurements made with Kepler on a large set of red giants have been compared in Mathur et al. (2011) with theoretical 
calculations performed on the basis of the Ludwig (2006) ab initio approach and using a grid of 3D hydrodynamical models of the surface layers of red giants computed with the STAGGER code (see a detailed description for instance in Trampedach 2004). Although these theoretical calculations reproduce the measured scaling relations rather well in terms of their timescale $\tau_{\text {eff }}$, and $\sigma$, large systematic differences were found with observations, however. While for the parameter $\tau_{\text {eff }}$ the dispersion to the scaling $v_{\max }^{-1}$ is very small, a more appreciable dispersion is observed for $\sigma$ with respect to the scaling $v_{\max }^{-1 / 2}$. Eventually, Mathur et al. (2011) calculations were limited to red giant stars. For main-sequence (MS) stars, such a modelling has been performed and the results compared with observations for a limited set of stars only (Trampedach et al. 1998; Svensson \& Ludwig 2005; Ludwig et al. 2009a; Guenther et al. 2008).

To justify the scaling relation between $\tau_{\text {eff }}$ and $v_{\mathrm{c}}$ (or equivalently $v_{\max }$ ), Huber et al. (2009) have conjectured that the granules move proportionally to the sound speed (see also Kjeldsen $\&$ Bedding 2011). The total brightness fluctuations $\sigma$ are known to scale as the inverse of the square root of the number of granules over half the stellar surface (see e.g. Ludwig 2006). This number in turn scales as $v_{\mathrm{c}} M / T_{\mathrm{eff}}^{3 / 2}$ (Kjeldsen \& Bedding 2011; Mathur et al. 2011), where $T_{\text {eff }}$ is the effective temperature and $M$ the mass of the star. However, $\sigma$ is expected to depend also on the intensity contrast of the granules and hence on their temperature contrast (see e.g. Ludwig et al. 2009a). Therefore, the theoretical scaling relations proposed for $\sigma$ and $\tau_{\text {eff }}$ (the "classical" scaling relations hereafter) still partially rely on some simplified physical assumptions and need to be completed.

Samadi et al. (2013, hereafter Paper I) proposed a theoretical model of the stellar granulation. This model predicts the power density spectrum associated with the relative variation of the disk-integrated flux due to the granulation at the surface of the star. Compared with the Ludwig (2006) ab initio approach, this theoretical model offers the advantage of testing separately several properties of the turbulent convection and can be extensively applied to a large set of stellar models. Our aim here is to derive theoretical scaling relations for $\tau_{\text {eff }}$ and $\sigma$ from this model and to compare them with the observations made by Kepler on a large set of targets. For this purpose, we applied the theoretical model of stellar granulation of Paper I to a set of 3D hydrodynamical models of the surface layers of stars with surface gravities ranging from $\log g=1.5$ to $\log g=4.5$ and effective temperatures ranging from $T_{\text {eff }} \simeq 6700 \mathrm{~K}$ (F-type star) to $T_{\text {eff }}=4000 \mathrm{~K}$ (K-type star). For each 3D model we computed the theoretical power density spectrum (PDS) associated with the granulation. From each spectrum we then extracted the characteristic time scale $\left(\tau_{\text {eff }}\right)$ and the brightness fluctuations $(\sigma)$ associated with the granulation in the same way as for the observations. We compare theses quantities with the theoretical scaling relations derived from the theoretical model and the characteristic times and brightness fluctuations extracted from Kepler targets.

\section{Theoretical model}

The theoretical model presented in Paper I aims at modelling the power density spectrum (PDS) associated with the relative variations of the bolometric flux emerging from the star in the direction of an observer and measured continuously during a given duration. Here we use the theoretical PDS as a function of the frequency $(v)$ given in Paper I

$\mathcal{F}(v)=\int_{0}^{1} \mathrm{~d} \mu \int_{0}^{+\infty} \mathrm{d} \tau \mathrm{e}^{-2 \tau / \mu}\left(\frac{\langle B\rangle_{t}}{F_{0}}\right)^{2} \mathcal{F}_{\tau}(\tau, v)$ with

$F_{0}=\int_{0}^{1} \mathrm{~d} \mu \int_{0}^{+\infty} \mathrm{d} \tau \mathrm{e}^{-\tau / \mu}\langle B\rangle_{t}(\tau)$

$\mathcal{F}_{\tau}(\tau, v)=2 \pi \tau_{\mathrm{c}} \sigma_{\tau}^{2} S_{\Theta}(\tau, v)$

$\sigma_{\tau}=\frac{12}{\sqrt{2}} \sqrt{\frac{\tau_{\mathrm{g}}}{\mathcal{N}_{\mathrm{g}}}} \Theta_{\mathrm{rms}}^{2}$

$\Theta_{\mathrm{rms}}=\frac{\Delta T_{\mathrm{rms}}}{T}$

$\tau_{\mathrm{g}}=\kappa \rho \Lambda$

$\mathcal{N}_{\mathrm{g}}=\frac{2 \pi R_{\mathrm{s}}^{2}}{\Lambda^{2}}$

where $B$ is the Planck function, $\tau$ the mean optical depth, $\kappa$ the mean opacity, $\Delta T_{\mathrm{rms}}$ the rms of the temperature fluctuations, $T$ and $\rho$ the stratification in temperature and density, respectively, $R_{\mathrm{S}}$ the stellar radius, $\Lambda$ the granule characteristic size, $\tau_{\mathrm{c}}$ the granule characteristic time, $\mu=\cos (\theta), \theta$ the angle between the direction pointing toward the observer and the direction normal to the stellar surface, the symbol \langle\rangle$_{t}$ stands for a time average, and finally, $S_{\Theta}$ is a dimensionless "source" function whose expression is given in Paper I.

The term $\mathcal{F}_{\tau}$ (Eq. (3)) in the integrand of Eq. (1) stands for the PDS of the granulation as it would be seen at the optical depth $\tau$. In Eqs. (2)-(7), $\tau_{\mathrm{g}}$ corresponds to the characteristic optical thickness of the granules, $\mathcal{N}_{\mathrm{g}}$ to the average number of granules distributed over half of the photosphere (i.e. at $r=R_{\mathrm{s}}$ ), and $\sigma_{\tau}$ to the rms brightnesses fluctuations associated with the granulation spectrum as one would see at the optical depth $\tau$.

The "source" function $S_{\Theta}$ requires a prescription for $\chi_{k}(v)$, the Fourier transform of the eddy-time correlation function (see Paper I). The best fit with the solar granulation spectrum was obtained when an exponential form was adopted for $\chi_{k}(v)$.

For comparison with the observations, we define $\sigma$ as the rms brightness fluctuations associated with the theoretical PDS $(\mathcal{F}(v))$. The latter satisfies by definition the Parseval-Plancherel relation

$\sigma^{2}=\int_{-\infty}^{+\infty} \mathrm{d} v \mathcal{F}(v)$.

Following Mathur et al. (2011), we consider $\tau_{\text {eff }}$ as the e-folding time associated with the auto-correlation function (ACF) of the relative flux variations due to the granulation. Note that since the ACF is also the Fourier transform (FT) of the PDS, it is thus obtained by computing numerically the FT of $\mathcal{F}(v)$.

\section{Theoretical PDS across the HR diagram}

\subsection{Grid of 3D hydrodynamical models}

The 3D models used in this work are taken from the CIFIST grid (Ludwig et al. 2009b) and have been computed with the $\mathrm{CO}^{5}$ BOLD code (Freytag et al. 2012). The adopted chemical mixture is similar to the solar chemical composition proposed by Asplund et al. (2005). Details about the CIFIST grid are given in Ludwig et al. (2009b). Of the 3D models of the CIFIST grid we considered only those with a solar metal abundance. Their characteristics are given in Table 1.

For comparison with the observations, it is convenient to introduce the peak frequency of the solar-like oscillations $\left(v_{\max }\right)$. This is shown to scale as the star's acoustic cut-off frequency, i.e. 
R. Samadi et al.: Stellar granulation as seen in disk-integrated intensity. II.

Table 1. Characteristics of the 3D hydrodynamical models and associated parameters (see text).

\begin{tabular}{|c|c|c|c|c|c|c|c|c|c|c|}
\hline Label & $\begin{array}{l}T_{\text {eff }} \\
{[\mathrm{K}]}\end{array}$ & $\begin{array}{c}\delta T_{\text {eff }} \\
{[\mathrm{K}]}\end{array}$ & $\begin{array}{c}\log g \\
{\left[\mathrm{~cm} / \mathrm{s}^{2}\right]}\end{array}$ & $\mathcal{M}_{\mathrm{a}}$ & $\begin{array}{l}\Theta_{\mathrm{rms}} \\
{[\%]}\end{array}$ & $\begin{array}{c}v_{\max } \\
{[\mu \mathrm{Hz}]}\end{array}$ & $\begin{array}{c}R_{\mathrm{S}} \\
{\left[R_{\odot}\right]}\end{array}$ & $\begin{array}{c}M \\
{\left[M_{\odot}\right]}\end{array}$ & $\begin{array}{c}\sigma \\
{[\mathrm{ppm}]}\end{array}$ & $\begin{array}{c}\tau_{\mathrm{eff}} \\
{[\mathrm{s}]}\end{array}$ \\
\hline $\mathrm{d} 3 \mathrm{t} 40 \mathrm{~g} 15 \mathrm{~mm} 00 \mathrm{n} 02$ & 4018 & 24 & 1.50 & 0.323 & 4.75 & 4.30 & 39.82 & 1.83 & $1.40 \times 10^{3}$ & $9.06 \times 10^{4}$ \\
\hline $\mathrm{d} 3 \mathrm{t} 45 \mathrm{~g} 25 \mathrm{~mm} 00 \mathrm{n} 01$ & 4476 & 10 & 2.50 & 0.274 & 3.72 & $4.07 \times 10^{1}$ & 10.85 & 1.36 & $3.17 \times 10^{2}$ & $1.09 \times 10^{4}$ \\
\hline $\mathrm{d} 3 \mathrm{t} 45 \mathrm{~g} 40 \mathrm{~mm} 00 \mathrm{n} 01$ & 4477 & 8 & 4.00 & 0.178 & 2.13 & $1.29 \times 10^{3}$ & 1.29 & 0.61 & $1.84 \times 10^{1}$ & $5.11 \times 10^{2}$ \\
\hline $\mathrm{d} 3 \mathrm{t} 48 \mathrm{~g} 32 \mathrm{~mm} 00 \mathrm{n} 01$ & 4775 & 12 & 3.20 & 0.260 & 3.45 & $1.98 \times 10^{2}$ & 4.89 & 1.38 & $1.11 \times 10^{2}$ & $2.49 \times 10^{3}$ \\
\hline $\mathrm{d} 3 \mathrm{t} 50 \mathrm{~g} 20 \mathrm{~mm} 00 \mathrm{n} 2$ & 4552 & 16 & 2.00 & 0.320 & 4.62 & $1.28 \times 10^{1}$ & 33.06 & 3.99 & $5.30 \times 10^{2}$ & $2.91 \times 10^{4}$ \\
\hline $\mathrm{d} 3 \mathrm{t} 50 \mathrm{~g} 25 \mathrm{~mm} 00 \mathrm{n} 01$ & 4969 & 18 & 2.50 & 0.378 & 4.66 & $3.86 \times 10^{1}$ & 18.25 & 3.76 & $3.44 \times 10^{2}$ & $1.01 \times 10^{4}$ \\
\hline $\mathrm{d} 3 \mathrm{t} 50 \mathrm{~g} 30 \mathrm{~mm} 00 \mathrm{n} 01$ & 5037 & 18 & 3.00 & 0.301 & 4.14 & $1.21 \times 10^{2}$ & 8.29 & 2.51 & $1.76 \times 10^{2}$ & $3.53 \times 10^{3}$ \\
\hline $\mathrm{d} 3 \mathrm{t} 50 \mathrm{~g} 35 \mathrm{~mm} 00 \mathrm{n} 01$ & 4924 & 13 & 3.50 & 0.245 & 3.17 & $3.88 \times 10^{2}$ & 3.53 & 1.44 & $6.94 \times 10^{1}$ & $1.32 \times 10^{3}$ \\
\hline $\mathrm{d} 3 \mathrm{t} 50 \mathrm{~g} 40 \mathrm{~mm} 00 \mathrm{n} 01$ & 4955 & 11 & 4.00 & 0.207 & 2.44 & $1.22 \times 10^{3}$ & 1.48 & 0.80 & $3.24 \times 10^{1}$ & $4.88 \times 10^{2}$ \\
\hline $\mathrm{d} 3 \mathrm{t} 50 \mathrm{~g} 45 \mathrm{~mm} 00 \mathrm{n} 04$ & 4981 & 12 & 4.50 & 0.172 & 1.98 & $3.86 \times 10^{3}$ & 0.85 & 0.83 & $1.13 \times 10^{1}$ & $1.77 \times 10^{2}$ \\
\hline $\mathrm{d} 3 \mathrm{t} 55 \mathrm{~g} 35 \mathrm{~mm} 00 \mathrm{n} 01$ & 5432 & 22 & 3.50 & 0.308 & 4.06 & $3.69 \times 10^{2}$ & 3.84 & 1.70 & $1.28 \times 10^{2}$ & $1.20 \times 10^{3}$ \\
\hline $\mathrm{d} 3 \mathrm{t} 55 \mathrm{~g} 40 \mathrm{~mm} 00 \mathrm{n} 01$ & 5476 & 13 & 4.00 & 0.257 & 3.30 & $1.16 \times 10^{3}$ & 1.64 & 0.98 & $6.45 \times 10^{1}$ & $4.47 \times 10^{2}$ \\
\hline $\mathrm{d} 3 \mathrm{t} 55 \mathrm{~g} 45 \mathrm{~mm} 00 \mathrm{n} 01$ & 5488 & 14 & 4.50 & 0.216 & 2.58 & $3.68 \times 10^{3}$ & 0.89 & 0.92 & $2.40 \times 10^{1}$ & $1.64 \times 10^{2}$ \\
\hline $\mathrm{d} 3 \mathrm{t} 59 \mathrm{~g} 35 \mathrm{~mm} 00 \mathrm{n} 01$ & 5885 & 16 & 3.50 & 0.381 & 4.39 & $3.55 \times 10^{2}$ & 3.84 & 1.70 & $2.02 \times 10^{2}$ & $1.11 \times 10^{3}$ \\
\hline $\mathrm{d} 3 \mathrm{t} 59 \mathrm{~g} 40 \mathrm{~mm} 00 \mathrm{n} 01$ & 5927 & 13 & 4.00 & 0.311 & 4.07 & $1.12 \times 10^{3}$ & 1.77 & 1.14 & $1.08 \times 10^{2}$ & $4.11 \times 10^{2}$ \\
\hline $\mathrm{d} 3 \mathrm{t} 59 \mathrm{~g} 45 \mathrm{~mm} 00 \mathrm{n} 01$ & 5861 & 25 & 4.50 & 0.257 & 3.35 & $3.56 \times 10^{3}$ & 0.96 & 1.07 & $3.90 \times 10^{1}$ & $1.53 \times 10^{2}$ \\
\hline $\mathrm{d} 3 \mathrm{t} 63 \mathrm{~g} 35 \mathrm{~mm} 00 \mathrm{n} 01$ & 6140 & 25 & 3.50 & 0.445 & 4.46 & $3.48 \times 10^{2}$ & 3.91 & 1.76 & $2.16 \times 10^{2}$ & $9.56 \times 10^{2}$ \\
\hline $\mathrm{d} 3 \mathrm{t} 63 \mathrm{~g} 40 \mathrm{~mm} 00 \mathrm{n} 02$ & 6227 & 16 & 4.00 & 0.362 & 4.38 & $1.09 \times 10^{3}$ & 1.93 & 1.36 & $1.25 \times 10^{2}$ & $3.77 \times 10^{2}$ \\
\hline $\mathrm{d} 3 \mathrm{t} 63 \mathrm{~g} 45 \mathrm{~mm} 00 \mathrm{n} 01$ & 6233 & 15 & 4.50 & 0.297 & 4.04 & $3.45 \times 10^{3}$ & 1.05 & 1.27 & $5.47 \times 10^{1}$ & $1.44 \times 10^{2}$ \\
\hline $\mathrm{d} 3 \mathrm{t} 65 \mathrm{~g} 40 \mathrm{~mm} 00 \mathrm{n} 01$ & 6486 & 20 & 4.00 & 0.416 & 4.46 & $1.07 \times 10^{3}$ & 1.99 & 1.44 & $1.37 \times 10^{2}$ & $3.54 \times 10^{2}$ \\
\hline $\mathrm{d} 3 \mathrm{t} 65 \mathrm{~g} 45 \mathrm{~mm} 00 \mathrm{n} 02$ & 6458 & 14 & 4.50 & 0.325 & 4.36 & $3.39 \times 10^{3}$ & 1.11 & 1.42 & $6.50 \times 10^{1}$ & $1.37 \times 10^{2}$ \\
\hline $\mathrm{d} 3 \mathrm{t} 68 \mathrm{~g} 43 \mathrm{~mm} 00 \mathrm{n} 01$ & 6725 & 17 & 4.25 & 0.406 & 4.35 & $1.87 \times 10^{3}$ & 1.45 & 1.36 & $9.92 \times 10^{1}$ & $2.00 \times 10^{2}$ \\
\hline $\mathrm{d} 3 \mathrm{gt} 57 \mathrm{~g} 44 \mathrm{n} 57$ & 5783 & 18 & 4.44 & 0.272 & 3.21 & $3.11 \times 10^{3}$ & 1.00 & 1.00 & $3.86 \times 10^{1}$ & $1.72 \times 10^{2}$ \\
\hline
\end{tabular}

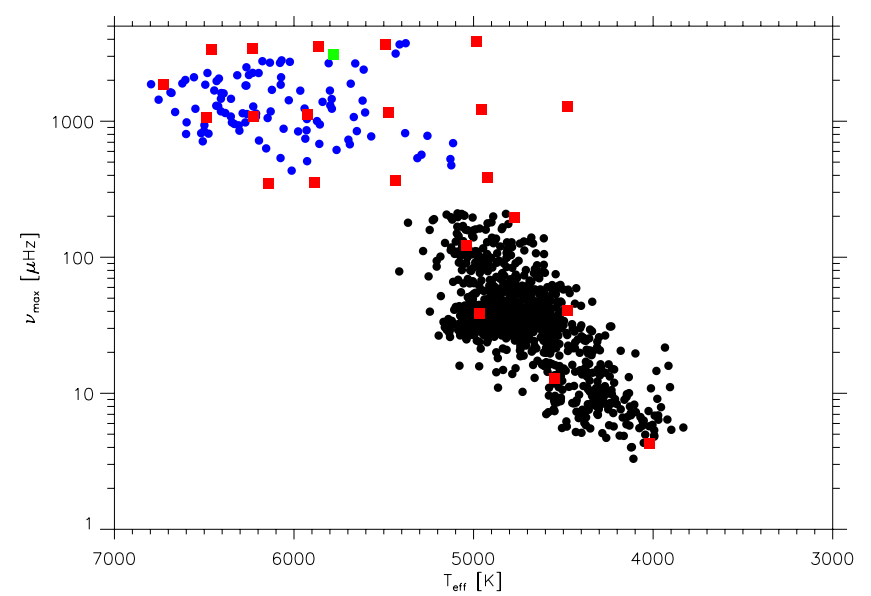

Fig. 1. $v_{\max }$ as a function of $T_{\text {eff }}$. The filled red squares correspond to the location of the 3D hydrodynamical models in the plan $v_{\max }-T_{\text {eff }}$ (see also Table 1), the filled blue circles to the Kepler sub-giant and MS targets, and the black ones to the Kepler red giants (see Sect. 5). The filled green square shows the position of our 3D solar model (the last model in Table 1).

as $g / \sqrt{T_{\text {eff }}}$ (Kjeldsen \& Bedding 1995; Stello et al. 2009; Huber et al. 2009; Mosser et al. 2010, 2013; Belkacem et al. 2011). Accordingly, we determined $v_{\max }$ for each $3 \mathrm{D}$ model with the following scaling:

$v_{\max }=v_{\text {ref }} \frac{g}{g_{\odot}} \sqrt{\frac{T_{\text {eff } \odot}}{T_{\text {eff }}}}$,

where $v_{\text {ref }}=3106 \mu \mathrm{Hz}$ (as in Mosser et al. 2013), $\log g_{\odot}=$ $4.438\left(\mathrm{~cm} / \mathrm{s}^{2}\right)$ and $T_{\text {eff }, \odot}=5777 \mathrm{~K}$. The values of $v_{\max }$ associated with each 3D model are given in Table 1 . The positions of the 3D models in the plane $T_{\text {eff }}-v_{\max }$ are displayed in Fig. 1.
To calculate the theoretical PDS we needed to know the radius of the star $\left(R_{\mathrm{s}}\right)$. We determined for each 3D model the associated radius using a grid of standard stellar models computed with the CESAM2k code (Morel \& Lebreton 2008). The stellar 1D models have the same chemical composition as the 3D models. The radius and mass associated with each $3 \mathrm{D}$ models are given in Table 1.

\subsection{Calculations of the theoretical PDS}

We computed the PDS of the granulation $(\mathcal{F})$ according to Eqs. (1)-(7). The different quantities involved in the theoretical model were obtained as detailed in Paper I from 3D hydrodynamical models of the surface layers of stars.

The theoretical model involves three free parameters: $\beta, \lambda$, and $\zeta$. The first controls the granule sizes, the second their characteristic time, and the last one the characteristic wave-number, which separates the inertial-convective range from the inertialconductive range associated with the spectrum of the temperature fluctuations (see details in Paper I). In Paper I we calibrated the three parameters using the observed solar granulation spectrum together with constraints from the resolved image of the solar granulation. The calibration gave $\lambda=0.30, \beta=3.42$ and $\zeta=5$ (see Paper I Sect. 3). These values were used for all calculations presented here.

For each 3D model, we computed the associated PDS and then derive the associated rms brightness fluctuation $\sigma$ (Eq. (8)) and the characteristic time $\tau_{\text {eff }}$ as defined in Sect. 2. Theoretical values of $\tau_{\text {eff }}$ and $\sigma$ are given in Table 1 and are compared with the observations in Sect. 6.

\section{Theoretical scaling relations}

It is observationally established (Kallinger \& Matthews 2010; Mathur et al. 2011; Chaplin et al. 2011b) that the characteristic time $\tau_{\text {eff }}$ associated with granulation varies from one star to 
another approximately as the inverse of the peak frequency of the solar-like oscillations $\left(v_{\max }\right)$. Brown et al. (1991) and Kjeldsen \& Bedding (1995) have conjectured that $v_{\max }$ scales as the star cutoff frequency, $v_{\mathrm{c}} \propto \mathrm{g} / \sqrt{T_{\mathrm{eff}}}$. This relation was shown to work for a larger variety of stars (Bedding \& Kjeldsen 2003; Stello et al. 2009; Huber et al. 2009; Mosser et al. 2010) and its underlying physical origin has been explained recently by Belkacem et al. (2011). For the rms brightness fluctuation $\sigma$, the observations show that $\sigma$ roughly scales as $\left(v_{\max }\right)^{-1 / 2}$ (Mathur et al. 2011; Chaplin et al. 2011b). As shown below, the theoretical model for the stellar granulation presented in Sect. 2 predicts the same dependence of $\sigma$ and $\tau_{\text {eff }}$ on $v_{\max }$. However, we will establish that $\sigma$ and $\tau_{\text {eff }}$ are also expected to scale as a function of the Mach number.

The integrand of Eq. (1) is highest close to the photosphere (i.e. around the optical depth $\tau=2 / 3$, or equivalently around $T=T_{\text {eff }}$ ). Therefore the rms brightness fluctuations $\sigma$ of the stellar granulation as well as its associated characteristic time $\tau_{\text {eff }}$ are closely controlled by $\tau_{\mathrm{c}}$ and $\sigma_{\tau}$ at the photosphere, where $\tau_{\mathrm{c}}$ and $\sigma_{\tau}$ are the granule life-time and the rms brightness fluctuation at the optical depth $\tau$ (see Sect. 2).

\subsection{Scaling for the characteristic time $\tau_{\text {eff }}$}

The characteristic time $\tau_{\mathrm{c}}$ is by definition proportional to $\Lambda / w_{\mathrm{rms}}$ (see Paper I), where $w_{\text {rms }}$ is the rms of the vertical component of the velocity. Let $\mathcal{M}_{\mathrm{a}}=w_{\mathrm{rms}} / c_{\mathrm{s}}$ be the turbulent Mach number, where $c_{\mathrm{s}}$ is the sound speed (both are evaluated at the photosphere). By hypothesis, $\Lambda$ varies as $H_{\mathrm{p}}$, which scales as $T_{\text {eff }} / g$. Furthermore, $c_{\mathrm{s}}$ varies at the photosphere as $\sqrt{T_{\text {eff }}}$. Accordingly, $\tau_{\text {eff }}$ is expected to scale as $\mathcal{M}_{\mathrm{a}} \sqrt{T_{\text {eff }}} /\left(g \mathcal{M}_{\mathrm{a}}\right) \propto\left(\mathcal{M}_{\mathrm{a}} v_{\mathrm{c}}\right)^{-1}$, and since $v_{\mathrm{c}} \propto v_{\max }$, we expect that

$$
\tau_{\text {eff }} \propto \frac{1}{\mathcal{M}_{\mathrm{a}} v_{\max }}
$$

\subsection{Scaling for the granulation amplitude $\sigma$}

The expression for $\sigma_{\tau}$ (Eq. (4)) involves three characteristic quantities:

- $\mathcal{N}_{\mathrm{g}}$ : the average number of granules over the stellar surface (Eq. (7));

- $\Theta_{\mathrm{rms}}=\Delta T_{\mathrm{rms}} / T:$ the rms of the (relative) temperature fluctuations;

- $\tau_{\mathrm{g}}$ : the optical thickness of the granules (Eq. (6)).

From Eq. (7) and the scaling relation for $\Lambda$ (see Sect. 4.1 above), one easily obtains that $\mathcal{N}_{\mathrm{g}}$ scales as $v_{\max } M T_{\mathrm{eff}}^{-3 / 2}$.

For $\Theta_{\text {rms }}$ we can derive the following relation between $w_{\text {rms }}$ and the relative temperature fluctuations (e.g. Cox 1968) following the mixing-length theory:

$w_{\mathrm{rms}}^{2}=g \Lambda \theta_{\mathrm{rms}}$.

Since $\Lambda \propto T / g$ and $c_{\mathrm{s}} \propto T^{1 / 2}$, Eq. (11) yields that $\Theta_{\text {rms }}$ scales as $\mathcal{M}_{\mathrm{a}}^{2}$. However, using a grid of stellar 3D models (see Sect. 3.1), we found that $\Theta_{\text {rms }}$ varies with $\mathcal{M}_{\mathrm{a}}$ in a more complicate manner. This is illustrated in Fig. 2 (top panel), where $\Theta_{\mathrm{rms}}$ is plotted as a function of $\mathcal{M}_{\mathrm{a}}$. Except for the two red-giant 3D models with $\log g \leq 2$ (see Table 1$), \Theta_{\text {rms }}\left(\mathcal{M}_{\mathrm{a}}\right)$ can be fitted very well by a second-order polynomial function

$\frac{\Theta_{\mathrm{rms}}}{\Theta_{0}} \equiv f\left(\mathcal{M}_{\mathrm{a}}\right)=a_{0}+a_{1}\left(\frac{\mathcal{M}_{\mathrm{a}}}{\mathcal{M}_{\mathrm{a}, 0}}\right)+a_{2}\left(\frac{\mathcal{M}_{\mathrm{a}}}{\mathcal{M}_{\mathrm{a}, 0}}\right)^{2}$,
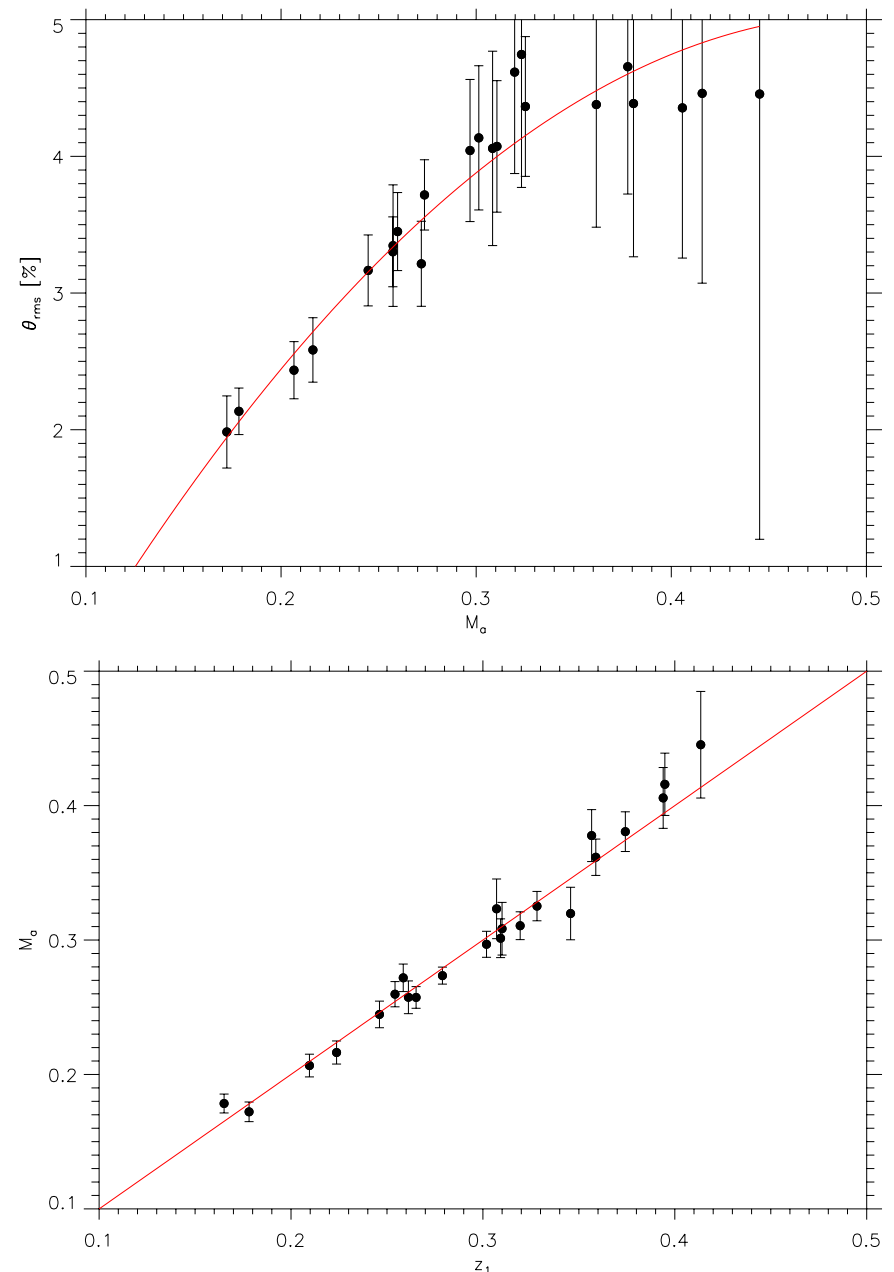

Fig. 2. Top: relative temperature fluctuation $\Theta_{\mathrm{rms}}$ (in \%) as a function of the Mach number $\mathcal{M}_{\mathrm{a}}$. The filled circles correspond to the values obtained for each 3D model (see Table 1). Theses values were computed at the photosphere (i.e. at the optical depth $\tau=2 / 3$ ). The red curve corresponds to the polynomial function given by Eq. (12). The two upper points that deviate most from the polynomial function correspond to the two 3D models with $\log g \leq 2$, i.e. the most evolved RG 3D models of our grid. Bottom: $\mathcal{M}_{\mathrm{a}}$ as a function of the quantity $z_{1}$ given by Eq. (17). The red line corresponds to a linear scaling in $z_{1}$.

where the coefficients $\left(a_{0}, a_{1}, a_{2}\right)$ are obtained by least-squares adjustment, and where we have defined the coefficients $\Theta_{0}=$ $3.2 \%$ and $\mathcal{M}_{\mathrm{a}, 0}=0.26$. The value $\Theta_{0}$ corresponds to the value associated with the solar 3D model (the last model listed in Table 1), while the value $\mathcal{M}_{\mathrm{a}, 0}=0.26$ is the one obtained by adjustment in Sect. 4.3. The fit yields $a_{0}=-0.67 \pm 0.21$, $a_{1}=2.30 \pm 0.43$ and $a_{2}=-0.59 \pm 0.22$ and is shown in Fig. 2 (top panel). It is interesting to note that Tremblay et al. (2013) have recently found a qualitatively similar dependence between the relative intensity contrast of the granules and $\mathcal{M}_{\mathrm{a}}$.

Finally, the optical thickness of the granule $\tau_{\mathrm{g}}$ is expected to vary slowly from one star to another. Indeed, we aim to evaluate this quantity near the photosphere, i.e. close to the unit optical depth. The optical thickness of the granule is by definition

$\tau_{\mathrm{g}}=\Lambda \kappa \rho=\beta H_{\mathrm{p}} \kappa \rho$,

where $\beta$ is the free parameter introduced in Paper I. The optical depth is given by $\tau(r)=\int_{r}^{+\infty} \mathrm{d} r \kappa \rho$. At the photosphere, the pressure scale-height, $H_{\mathrm{p}}$, is of the same order as the density 
scale height $H_{\rho}$. If we assume that the opacity and the density scale height vary slowly in the atmosphere, we obtain that at a given optical depth $\tau \propto \kappa \rho H_{\mathrm{p}} \propto \tau_{\mathrm{g}}$. The photosphere corresponds by definition to the optical depth $\tau=2 / 3$. Therefore, if we evaluate $\tau_{\mathrm{g}}$ at the photosphere, we then expect that $\tau_{\mathrm{g}}$ remains constant from a star to another.

When we combine the scaling relations found for $\mathcal{N}_{\mathrm{g}}$ and $\Theta_{\mathrm{rms}}$, we find the following scaling:

$\sigma \propto \frac{f^{2}\left(\mathcal{M}_{\mathrm{a}}\right)}{v_{\max }^{1 / 2}} \frac{T_{\mathrm{eff}}^{3 / 4}}{M^{1 / 2}}$,

where the function $f\left(\mathcal{M}_{\mathrm{a}}\right)$ is given by Eq. (12). The term $T_{\text {eff }}^{3 / 4} M^{-1 / 2} v_{\max }^{-1 / 2}$ in Eq. (14) corresponds to the classical scaling relation (Kjeldsen \& Bedding 2011; Mathur et al. 2011), and comes basically from the fact that $\sigma$ scales as the inverse of the square root of the number of granules over the stellar surface (see e.g. Ludwig 2006). Our theoretical model then consistently results in the same dependence as the classical scaling relation. However, we find here that $\sigma$ also scales with a function of the Mach number. It is worth noting that for MS stars, the term $\left(T_{\mathrm{eff}}^{3 / 4} / M^{1 / 2}\right)$ varies slowly. Indeed, according to Noyes et al. (1984), $M \propto T^{1.8}$ for MS stars.

\subsection{Scaling for the mach number $\mathcal{M}_{\mathrm{a}}$}

To compare the scaling relations established for $\tau_{\text {eff }}$ (Eq. (10)) and $\sigma$ (Eq. (14)) with the observations, it is necessary to derive the Mach number $\mathcal{M}_{\mathrm{a}}$ as a function of some fundamental parameters of the star. As a guideline, we first derived such a scaling on the basis of simple physical assumptions. We then derived a more appropriate scaling using the grid of 3D models.

We first established a scaling for the flux of kinetic energy $F_{\text {kin }} \approx \rho w_{\text {rms }}^{3}$. In the framework of the mixing-length approach, it can be shown that $F_{\text {kin }}$ is roughly proportional to the convective flux $F_{\mathrm{c}}$. In the layer where the granulation is observed, the total energy flux, $F_{\text {tot }}$, is no longer transported dominantly by convection. However, to derive an expression for $F_{\text {kin }}$ that depends only on the surface parameters of the star, we assumed that the entire energy is transported by convection; that is $F_{\mathrm{c}} \approx F_{\text {tot }}=\sigma T_{\text {eff }}^{4}$, where $\sigma$ is the Stefan-Boltzmann constant. Accordingly, at the photosphere we have $\mathcal{M}_{\mathrm{a}} \equiv w_{\mathrm{rms}} / c_{\mathrm{s}} \propto T_{\text {eff }}^{5 / 6} \rho_{\mathrm{s}}^{-1 / 3}$ where $\rho_{\mathrm{s}}$ is the density at the photosphere.

We now need to establish a scaling for $\rho_{\mathrm{s}}$. As done in Sect. 4.2, we approximated the optical depth as $\tau \approx \kappa \rho H_{\mathrm{p}}$. Since our goal is to derive the scaling relation for $\mathcal{M}_{\mathrm{a}}$ at the photosphere, which by definition is such that $\tau=2 / 3$, we then obtain that $\rho_{\mathrm{s}}$ must scale as $g T_{\mathrm{eff}}^{-1} \kappa^{-1}$.

For the low-mass stars we are interested in, which have typical temperatures ranging between 4000 and $6000 \mathrm{~K}$, the dominant opacity source is $\mathrm{H}^{-}$. Using tables, it has been possible to show that the related opacity follow the power law (e.g. Hansen \& Kawaler 1994)

$\kappa \propto \rho^{1 / 2} T^{9}$.

We finally establish

$\mathcal{M}_{\mathrm{a}} \propto z_{0} \equiv\left(\frac{T_{\mathrm{eff}}}{T_{\mathrm{eff}, \odot}}\right)^{3}\left(\frac{g}{g_{\odot}}\right)^{-2 / 9}$.

Guided by this scaling, we fitted the following analytical expression on our set of values of $\mathcal{M}_{\mathrm{a}}$ :

$\mathcal{M}_{\mathrm{a}}=z_{1} \equiv\left(\frac{T_{\mathrm{eff}}}{T_{\mathrm{eff}, \odot}}\right)^{a}\left(\frac{g}{g_{\odot}}\right)^{-b} \mathcal{M}_{\mathrm{a}, 0}$, where $a, b$ and $\mathcal{M}_{\mathrm{a}, 0}$ are coefficients obtained by least-squares adjustment. The best fit is obtained with $a=2.35 \pm 0.09$, $b=0.152 \pm 0.007$ and $\mathcal{M}_{\mathrm{a}, 0}=0.258 \pm 0.003$. The overall satisfactory agreement of the scaling given by Eq. (17) with the individual values for $\mathcal{M}_{\mathrm{a}}$ (Table 1) is illustrated in Fig. 2 (bottom panel).

\subsection{Comparison with individual theoretical values}

We compared the theoretical scaling relations derived for $\tau_{\text {eff }}$ (Sect. 4.1) and $\sigma$ (Sect. 4.2) with the individuals values obtained with our grid of 3D models (see Sect. 3 and Table 1) as well as with the classical scaling relations.

We started with the scaling relations for $\tau_{\text {eff }}$. We recast the scaling relation given by Eq. (10) as

$\tau_{\mathrm{eff}} \propto z_{2} \equiv\left(\frac{v_{\mathrm{ref}}}{v_{\mathrm{max}}}\right)\left(\frac{\mathcal{M}_{\mathrm{a}, 0}}{\mathcal{M}_{\mathrm{a}}}\right)$

We have plotted in Fig. 3 (top panel) individual theoretical values of $\tau_{\text {eff }}$ (red squares) as a function of the quantity $z_{2}$. The individual theoretical values of $\tau_{\text {eff }}$ (Table 1 ) were found to scale as $z_{2}^{p}$ with $p=0.98$. This scaling is then close from the theoretical scaling relation $z_{2} \propto\left(v_{\max } \mathcal{M}_{\mathrm{a}}\right)^{-1}$. However, there is a slow deviation from a linear scaling with $z_{2}$, which must very likely be attributed to the various simplifications adopted in Sect. 4.1 to derive this scaling relation. For comparison with the classical scaling relation for $\tau_{\text {eff }}$, we defined the quantity $c_{2} \equiv\left(\nu_{\text {ref }} / v_{\max }\right)$. We have plotted in Fig. 3 (top panel) individual theoretical values of $\tau_{\text {eff }}$ (black circles) as a function of $c_{2}$. Theoretical $\tau_{\text {eff }}$ are found to scale as $c_{2}^{n}$ with $n=0.94$. The deviation from a linear scaling were therefore higher than with the scaling with $z_{2}$. Furthermore, for MS and sub-giant stars a much higher dispersion is obtained than with the new scaling relation. This shows that for these stars the Mach number significantly influences the characteristic time $\tau_{\text {eff }}$. On the other hand, for evolved stars, the variation of $\tau_{\text {eff }}$ is dominated by the variation of $v_{\max }$ along the evolution, which is basically related to rapid variation of the surface gravity (or equivalently the luminosity).

We turn now to the scaling relations for $\sigma$. The theoretical scaling relation given by Eq. (14) is recast as

$\sigma \propto z_{3} \equiv\left(\frac{T_{\mathrm{eff}}}{T_{\mathrm{eff}, \odot}}\right)^{3 / 4}\left(\frac{M_{\odot}}{M}\right)^{1 / 2}\left(\frac{v_{\mathrm{ref}}}{v_{\max }}\right)^{1 / 2}\left(\frac{f\left(\mathcal{M}_{\mathrm{a}}\right)}{f\left(\mathcal{M}_{\mathrm{a}, 0}\right)}\right)^{2}$.

As seen in Fig. 3 (bottom panel), individual theoretical values of $\sigma$ (red squares) vary globally according to the theoretical scaling relation given by Eq. (19). The scaling of $\sigma$ with $z_{3}$ is not fully linear since a fit gives $\sigma \propto z_{3}^{p}$ with $p=1.10$ (see Fig. 3, bottom panel). This deviation from a linear scaling with $z_{3}$ is shown in Appendix B to arise for a large part from the considerable degeneracy between $R$ and $M$ that occurs for RG stars. For comparison with the classical scaling relation, we defined the quantity $c_{3}$ as

$c_{3} \equiv\left(\frac{T_{\mathrm{eff}}}{T_{\mathrm{eff}, \odot}}\right)^{3 / 4}\left(\frac{M_{\odot}}{M}\right)^{1 / 2}\left(\frac{v_{\mathrm{ref}}}{v_{\max }}\right)^{1 / 2}$.

Theoretical values of $\sigma$ are plotted in Fig. 3 (bottom panel) as a function of $c_{3}$. Again, for the MS and sub-giant stars, a considerable dispersion is obtained w.r.t. the classical scaling relation $c_{3}$. The dispersion is substantially reduced when $\sigma$ is plotted as a function of the new scaling relation $\left(z_{3}\right)$. 

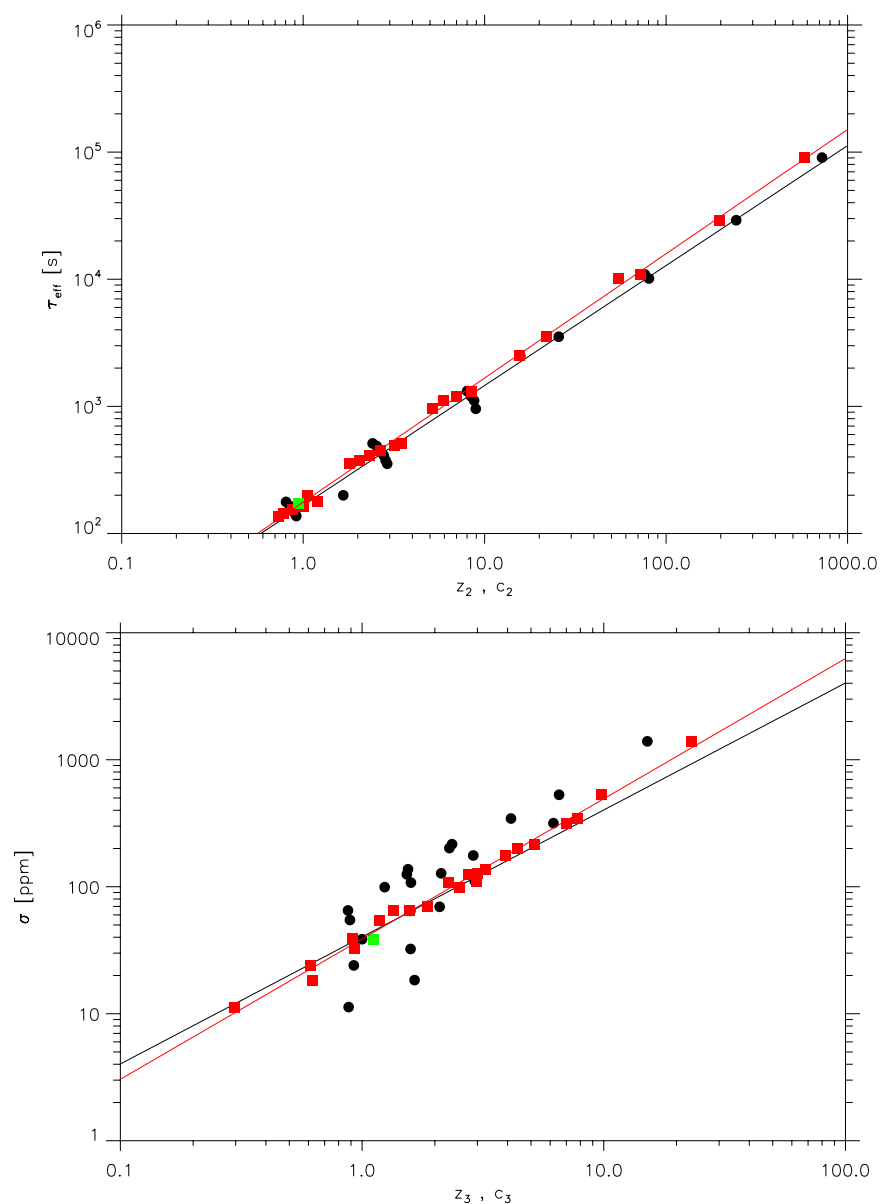

Fig. 3. Top: theoretical values of $\tau_{\text {eff }}$ as a function of the quantity $z_{2}$ (red squares) given by Eq. (18) and as a function of the classical scaling $c_{2} \equiv\left(v_{\text {ref }} / v_{\max }\right)$ (filled black circles). The symbols correspond to the individual theoretical values obtained with our grid of 3D models. The red curve corresponds to a power law of the form $z_{2}^{p}$ where the slope $p=0.98$ is obtained by fitting the red squares, while the black line is a power law of the form $c_{2}^{n}$ where the slope $n=0.94$ is obtained by fitting the black circles. Bottom: theoretical values $\sigma$ as a function the quantity $z_{3}$ given by Eq. (19) and as a function of the classical scaling $c_{3}$ (Eq. (20)). The symbols correspond to the individual theoretical values obtained with our grid of 3D models. The red curve is a power law of the form $z_{3}^{p}$ where the slope $p=1.10$ is obtained by fitting the red squares, while the black line is a linear scaling in $c_{3}$.

As a conclusion, despite the simplifications adopted for deriving the new scaling relations, they are found to match the values of $\sigma$ and $\tau_{\text {eff }}$ derived from the theoretical PDS reasonably well. On the other hand, the classical scaling relations significantly departs from the theoretical values.

\section{Observations}

Characteristics of stellar granulation in terms of time-scale $\left(\tau_{\text {eff }}\right)$ and rms brightness fluctuations $(\sigma)$ are typically extracted from the power spectrum of the intensity using various background models. Different analysis methods are found in the literature. They mainly differ from each other in 1) the number of components fitted in addition to the granulation component (e.g. activity, super-granulation, modes, misidentified components), and 2) the functional forms adopted for each component.

A major source of uncertainty related to those methods arises from the kink that is more or less visible on the stellar background. Such a feature has been first identified in the power spectrum of the solar irradiance data from the SOHO/VIRGO instrument at around $1 \mathrm{mHz}$ (Andersen et al. 1998; Vázquez Ramió et al. 2005). Similar features seem to be observed by Kepler on RG stars (Mathur et al. 2011). Furthermore, Karoff et al. (2013) recently analysed three MS stars for which this kink is visible and statistically significant. The physical origins of this kink are subjects of debates. They are either attributed to the occurrence of bright points (e.g. Harvey et al. 1993; Aigrain et al. 2004), the changing properties of the granules (Andersen et al. 1998), a second granulation population (Vázquez Ramió et al. 2005), or to faculae (Karoff 2012).

Because the origin of this second component is not yet clear, and it is furthermore missing in the current theoretical models, we considered the high-frequency component to be part of the granulation spectrum and compared the characteristics of the whole granulation spectrum with our theoretical predictions.

To compare measured values of $\sigma$ and $\tau_{\text {eff }}$ with theoretical calculations, we fitted the observed stellar backgrounds with the same functional form for all types of stars (MS stars to red giants). Of the different forms studied in Mathur et al. (2011), the Lorentzian function (also named in this context the Harvey model, Harvey 1985) results in values for $\tau_{\text {eff }}$ and the height of the granulation spectra that are enclosed by the different other methods of analysis investigated by Mathur et al. (2011). Accordingly, we adopted a Lorentzian function as in Mathur et al. (2011) as a reference for our comparisons with the theoretical calculations,

$\mathcal{P}(v)=\frac{H_{\mathrm{g}}}{1+\left(2 \pi \tau_{\mathrm{eff}} v\right)^{2}}$,

where the height $H_{\mathrm{g}}$ and $\tau_{\mathrm{eff}}$ were obtained as explained below. Note that prior to the calculation and the fit of the PDS, all light-curves were corrected following the procedures described in García et al. (2011).

For red giants, $H_{\mathrm{g}}$ and $\tau_{\mathrm{eff}}$ were determined with the method named "COR" in Mathur et al. (2011) that was also used in Mosser et al. (2012). Locally around $v_{\max }$ in a frequency range equal to [0.15-6] $\times v_{\max }$, one Lorentzian component is enough for fitting the background. The scaling relation $\tau_{\text {eff }} \propto v_{\max }^{-1}$ and the determination of the background $B_{\max }$ at $v_{\max }$ provided guess values of $\tau_{\text {eff }}$ and $H_{\mathrm{g}}$. The seismic excess energy was modelled with a Gaussian with a FWHM and an amplitude that are also governed by scaling relations (e.g. Mosser et al. 2010; Hekker et al. 2011). All parameters were then iteratively determined. The sample of red giants from which $H_{\mathrm{g}}$ and $\tau_{\text {eff }}$ were extracted was the same as in Mosser et al. (2012) or in Mathur et al. (2011).

More than about 500 of the sub-giants and MS stars, observed during Kepler's survey phase (i.e., based on one month of observations) were seen to be oscillating by Kepler. Here, however, we have analysed a cohort of the global sample that have been selected for long-term follow-ups and have thus been observed for at least three months from Quarter 5 onwards. This cohort initially contained 196 stars. In our final cut, we have retained the results from 141 stars. Taking $v_{\max } \approx 800 \mu \mathrm{Hz}$ as the threshold separating sub-giant from MS stars, 108 targets of the 141 targets of the sample can thus be considered as MS stars. Our analysis of their stellar backgrounds assumed a single Harvey-like profile (cf. Eq. (21)) describing granulation, a flat component describing shot noise, and a Gaussian envelope describing the p-mode hump. The power spectra were fitted in a range starting at $100 \mu \mathrm{Hz}$ and extending to the Nyquist frequency of Kepler short-cadence data $(8.5 \mathrm{mHz})$. A maximum-likelihood 
approach was employed to determine $H_{\mathrm{g}}$ and $\tau_{\text {eff }}$. More details about this analysis can be found in Karoff et al. (2013).

The rms brightness fluctuations $\sigma$, associated with the form given by Eq. (21) was obtained according to the relation $\sigma^{2}=$ $C_{\text {bol }}^{2} H_{\mathrm{g}} / \tau_{\text {eff }} / 4$, where $C_{\text {bol }}$ is a bolometric correction that scales for the Kepler bandpass as $C_{\text {bol }}=\left(T_{\text {eff }} / T_{0}\right)^{\alpha}$, where $T_{0}=5934 \mathrm{~K}$ and $\alpha=0.8$ (Ballot et al. 2011, see also Michel et al. 2009).

Measured values of $\tau_{\text {eff }}$ and $\sigma$ are compared in the next section with those obtained from the theoretical PDS, as explained in Sect. 2. However, in this comparison one must keep in mind that our measured values of $\tau_{\text {eff }}$ and $\sigma$ depend on the way the stellar background is modelled, in particular, whether or not the high-frequency component is included in the background model. As mentioned above, relative uncertainties of about $30 \%$ (peakto-peak) remain.

\section{Comparison with the observations}

\subsection{Characteristic time-scale, $\tau_{\text {eff }}$}

First, we compare in Fig. 4 (top panel) individual theoretical values of $\tau_{\text {eff }}$ (red squares) with measured ones. Sub-giants and MS stars overlap the theoretical and measured values of $\tau_{\text {eff }}$. However, for red-giant stars, the theoretical $\tau_{\text {eff }}$ systematically underestimates the measurements by about $40 \%$. This discrepancy is of the same order as that obtained by Mathur et al. (2011). Our theoretical $\tau_{\text {eff }}$ scales as $v_{\max }^{p}$ with $p=-0.94$, which agrees with Mathur et al. (2011). Indeed, their average value of the slope is $p=-0.89$ with a maximum difference of 0.03 between the different methods. In both cases, however, $\tau_{\text {eff }}$ significantly departs from a linear scaling with $v_{\max }$.

Second, to compare the theoretical scaling relation in $\left(v_{\max } \mathcal{M}_{\mathrm{a}}\right)^{-1}$ (Eq. (18)) with the observations, we used the scaling relation found for $\mathcal{M}_{\mathrm{a}}$ (Eq. (17)). This requires knowing $T_{\text {eff }}$ and the gravity $g$. For RG stars, the effective temperatures of the targets were obtained from the Kepler Input Catalogue (Brown et al. 2011) and corrected following Thygesen et al. (2012). For 136 of 141 sub-giant and MS targets, the effective temperatures were obtained from the Sloan Digital Sky Survey (Pinsonneault et al. 2012). For the five remaining targets, they were extracted from Silva Aguirre et al. (2012). The surface gravity $g$ of the targets were determined using the scaling relation for $v_{\max }$ (Eq. (9)).

We have plotted in Fig. 4 (bottom panel) measured and theoretical values of $\tau_{\text {eff }}$ as a function of the quantity $z_{2}$ given by Eq. (18). The measured values of $\tau_{\text {eff }}$ are found to scale as $z_{2}^{m}$ with $m=1.01$, which is very close to the expected theoretical scaling in $z_{2} \propto\left(v_{\max } \mathcal{M}_{\mathrm{a}}\right)^{-1}$ and the departure from a linear scaling is lower than that observed with the classical scaling (i.e. with $\left.c_{2} \propto v_{\max }^{-1}\right)$. However, the theoretical $\tau_{\text {eff }}$ systematically underestimates the observations by about $40 \%$. A large part of this systematic difference is a consequence of our choice of fitting the granulation background with a Lorentzian function (Eq. (21)).

The new theoretical scaling for $\tau_{\text {eff }}$ reproduces the observations on a global scale. However, the question remains whether or not the observations allow one to quantitatively confirm the dependence on $\mathcal{M}_{\mathrm{a}}$. We investigate this question in Appendix A.1 and conclude that the Kepler observations cannot distinguish the new scaling relation from the classical one.

\subsection{Brightness fluctuation $\sigma$}

The theoretical values of $\sigma$ are compared in Fig. 5 (top panel) with the measured ones as a function of $v_{\max }$. As in Mathur et al. (2011), our theoretical values of $\sigma$ scale approximately as $v_{\max }^{-1 / 2}$,
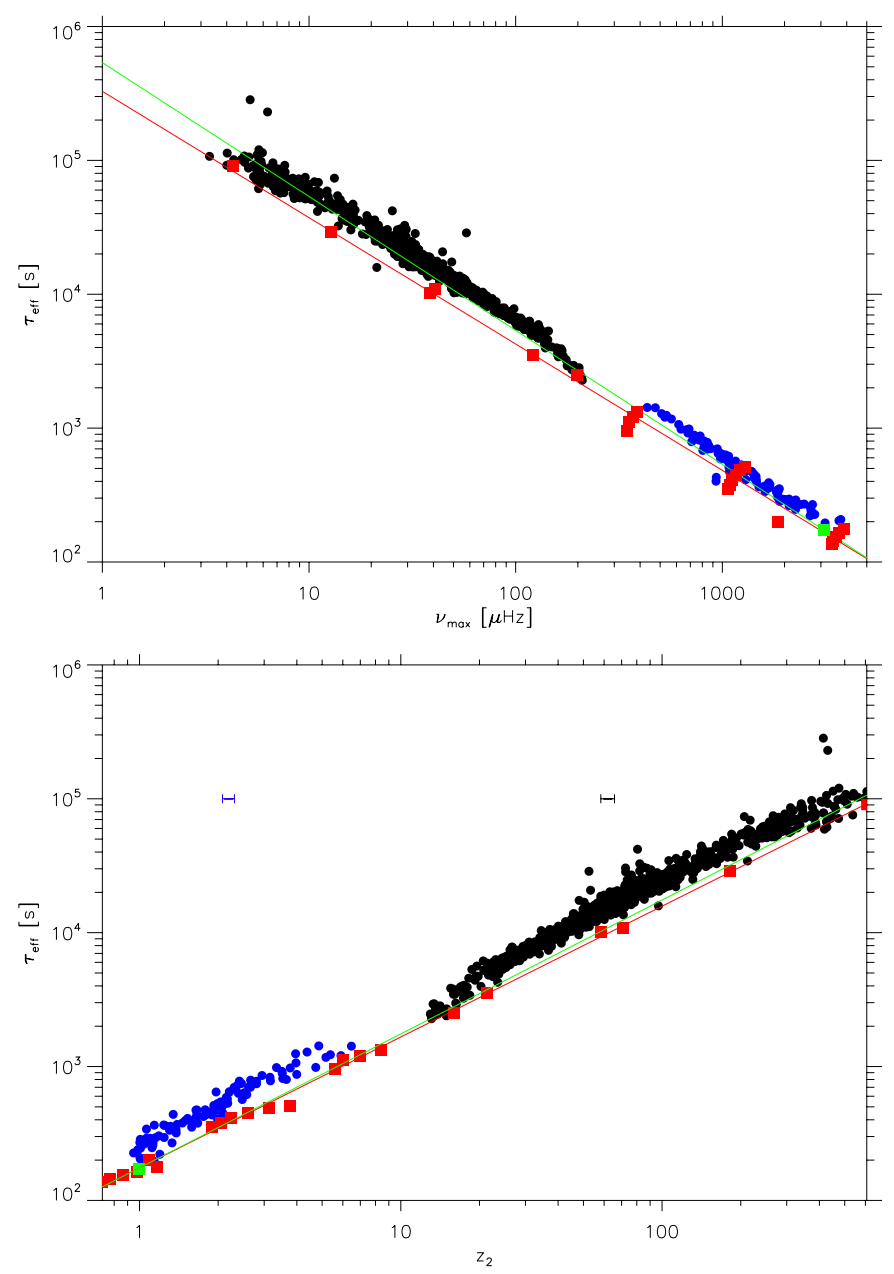

Fig. 4. Top: characteristic time $\tau_{\text {eff }}$ as a function of $v_{\max }$. The dots have the same meaning as in Fig. 1. The green curve correspond to a linear scaling in $v_{\max }^{-1}$ while the red curve is a power law of the form $v_{\max }^{p}$ with the slope $p=-0.94$ obtained by fitting the power law to the theoretical values of $\tau_{\text {eff }}$ (filled red squares). The filled green square corresponds to the value $\tau_{\text {eff }}=173 \mathrm{~s}$ found for the solar $3 \mathrm{D}$ model (the last model in Table 1). Bottom: $\tau_{\text {eff }}$ as a function of the scaling $z_{2}=\left(v_{\max } / v_{\mathrm{ref}}\right)\left(\mathcal{M}_{\mathrm{a}} / \mathcal{M}_{\mathrm{a}, 0}\right)$ where $\mathcal{M}_{\mathrm{a}}$ is supposed to scale according to Eq. (17). The symbols have the same meaning as in Fig. 1. The green line corresponds to a linear scaling in $z_{2}$, while the red curve to a power law of the form $z_{2}^{p}$ where the slope $p=0.98$ is obtained by fitting the theoretical values of $\tau_{\text {eff }}$ (red squares). The horizontal error bars show the uncertainty in $z_{2}$ associated with a typical uncertainty of $100 \mathrm{~K}(\mathrm{rms})$ in $T_{\text {eff. }}$. The blue horizontal error bar corresponds to a typical MS and the black one to a typical RG star.

in agreement with the observations. For RG stars, theoretical $\sigma$ falls within the observational domain. However, the dispersion in the theoretical calculations is much lower than in the measurements. This dispersion in the measurements must be linked to the fact that we observed a sample of stars inhomogeneous in terms of surface metal abundance.

For sub-giant and MS stars the observations and the theoretical values overlap. However, at fixed values of $v_{\max }$, theoretical values of $\sigma$ extend over a wider range than the observations. This is in part because the theoretical calculations include 3D models corresponding to dwarf stars cooler than the observed targets. Indeed, as seen in Fig. 1, sub-giant and MS stars cooler than about $5000 \mathrm{~K}$ and with $\nu_{\max } \gtrsim 800 \mu \mathrm{Hz}$ are lacking in our sample. According to our calculations, these stars are expected to 

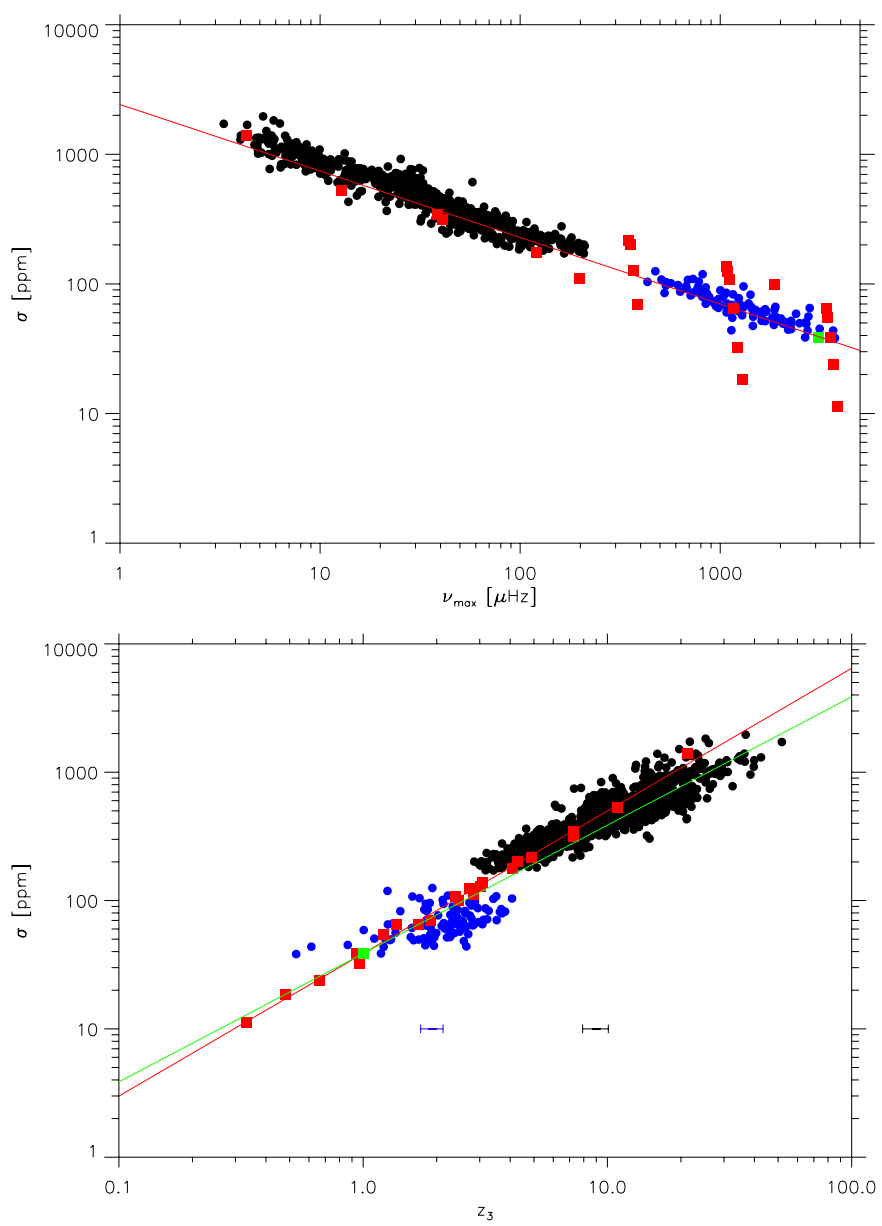

Fig. 5. Top: root-mean-square brightness fluctuation $\sigma$ as a function of $v_{\max }$. The symbols have the same meaning as in Fig. 1 . The red curve is a power law of the form $v_{\max }^{p}$ with the slope $p=-0.51$ obtained by fitting the power law to the theoretical values of $\sigma$ (filled red squares). The filled green square corresponds to the value $\sigma=39 \mathrm{ppm}$ found for the solar 3D model. Bottom: $\sigma$ as a function of the quantity $z_{3}$ given by Eq. (19) where $\mathcal{M}_{\mathrm{a}}$ and $T_{\text {eff }}^{3 / 4} M^{-1 / 2}$ are here supposed to scale according to Eq. (17) and Eq. (22), respectively. The green line corresponds to a linear scaling with $z_{3}$ and the red one to a power law of the form $z_{3}^{p}$ where $p=1.11$.

have a value of $\sigma$ about one to two orders of magnitude lower than observed.

We now compare the theoretical scaling given by Eq. (19) with the observations. The Mach number is estimated using Eq. (17), while the term $T_{\mathrm{eff}}^{3 / 4} M^{-1 / 2}$ can be derived using the scaling relations associated with $v_{\max }$ and the large separation $\Delta v$ (see e.g. Stello et al. 2009; Kallinger et al. 2010; Mosser et al. 2010, for a recent review see Belkacem et al. 2013). Indeed, combining the two seismic relations yields

$\frac{T_{\mathrm{eff}}^{3 / 4}}{M^{1 / 2}}=\left(\frac{v_{\mathrm{max}}}{v_{\mathrm{ref}}}\right)^{-3 / 2}\left(\frac{\Delta v}{\Delta v_{\mathrm{ref}}}\right)^{2} \frac{T_{\mathrm{eff}, \odot}^{3 / 4}}{M_{\odot}^{1 / 2}}$.

In Fig. 5 (bottom panel), we have plotted the measured values $\sigma$ as a function of $z_{3}$ (Eq. (19)) where $\mathcal{M}_{\mathrm{a}}$ and the term $T_{\mathrm{eff}}^{3 / 4} M^{-1 / 2}$ are here evaluated according to the scaling relations given by Eq. (17) and Eq. (22), respectively. The measured values of $\sigma$ are generally aligned with the linear relation in $z_{3}$.

Similarly to the procedure adopted for the scaling relation for $\tau_{\text {eff }}$, we investigate in Appendix A.2 the question whether or not the current observations can distinguish the new scaling relation from the classical one. It is found that compared with the new scaling relation, the classical one results in a smaller difference with the observations. However, the deviations of both scaling relations from the measurements are found to depend on $T_{\text {eff }}$, and the highest deviations are obtained for the F-dwarf stars $\left(T_{\text {eff }}=6000-7500 \mathrm{~K}\right)$. As discussed in Sect. 7, this is very likely a consequence of the lack of modelling of the impact of magnetic activity on the granulation background. Indeed, a high level of magnetic activity is expected to inhibit the surface convection and consequently reduce the Mach number. As stressed in Paper I, our theoretical calculations must be rigorously valid for stars with a low level of activity. If we now exclude the F-dwarf stars from our sample, we find that the current observations do not allow us to distinguish the new theoretical scaling relation from the classical one. It is finally established that only cool K-dwarf stars will allow us in principle to distinguish the dependence of $\sigma$ on $\mathcal{M}_{\mathrm{a}}$.

\section{Activity and granulation background}

Chaplin et al. (2011a) have shown clear evidence that a high level of magnetic activity inhibits the amplitudes of the solarlike oscillations and the strongest effects were - on average observed for F-dwarfs (Chaplin et al. 2011b). The authors concluded that this constitutes strong evidence for the impact of the magnetic activity on the near-surface convection. In this context, the case of the F-dwarf star HD 49933 is particularly enlightening. Indeed, this star shows clear evidence of a high level of activity (see Mosser et al. 2005, 2009; García et al. 2010). Ludwig et al. (2009a) have compared the theoretical granulation spectrum computed on the basis of the ab initio approach (Ludwig et al. 2009a) with the one measured with CoRoT on HD 49933. Their theoretical calculation results in an overestimation of the measured $\sigma$ by about $70 \%$, however. The authors argued that such a discrepancy is common to the F-dwarfs observed by CoRoT.

Consistently with the Ludwig et al. (2009a) results, our theoretical calculations result in a trend towards a high overestimation of the measured $\sigma$ and a moderate underestimation of $\tau_{\text {eff }}$ for F-dwarf stars. This is illustrated for $\sigma$ in Fig. 6, where we have plotted the relative differences $D_{\sigma}$ and $D_{\sigma}^{\prime}$ as a function of $T_{\text {eff }}$ (see Appendix A.2). The strongest relative differences are obtained around $T_{\text {eff }} \approx 6400 \mathrm{~K}$. On the other hand, the weakest differences are observed on average with stars cooler than about $6000 \mathrm{~K}$. This observed trend is very likely due to the impact of the magnetic activity on the granulation background, which is not included in the current modellings. Indeed, a high level of magnetic activity can inhibit the surface convection to some extent (see e.g. Nordlund et al. 2009, and references therein) and consequently inhibit the granulation background.

We note that reducting the photospheric Mach number of the F-dwarf stars by about $30 \%$ leads on average to values of $\sigma$ and $\tau_{\text {eff }}$ close to the measurements. Reduced thus, the Mach number is at about the same level as that of the solar $3 \mathrm{D}$ model $\left(\mathcal{M}_{\mathrm{a}} \simeq 0.27\right)$. For instance, the unexpectedly low observed amplitude measured for HD 49933 can been explained if we adopt for this star $\mathcal{M}_{\mathrm{a}}=0.30$, which is about $30 \%$ lower than predicted for the F-dwarf 3D model representative for HD 49933. However, whether or not magnetic activity can indeed reduce $\mathcal{M}_{\mathrm{a}}$ by this amount remains an open question.

The classical scaling relation also shows an excess around $T_{\mathrm{eff}} \approx 6400 \mathrm{~K}$. However, this is much weaker than for the new scaling relation. Given the fact that the classical scaling relation 


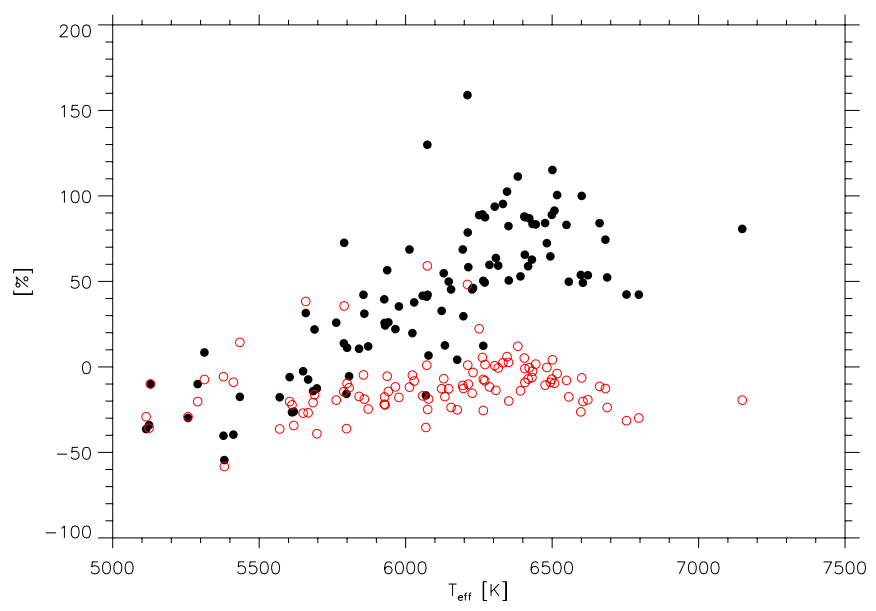

Fig. 6. Relative differences (in $\%$ ) between the theoretical $\sigma$ and the measured ones as a function of $T_{\text {eff }}$. The black filled circles correspond to the relative difference $D_{\sigma}=\left(\sigma_{\odot} / \sigma_{\mathrm{m}}\right) z_{3}-1$ and the red open circles to the relative difference $D_{\sigma}^{\prime}=\left(\sigma_{\odot} / \sigma_{\mathrm{m}}\right) c_{3}-1$, where $z_{3}$ (Eq. (19)) is the new theoretical scaling relation, $c_{3}$ (Eq. (20)) is the classical one, and $\sigma_{\mathrm{m}}$ the measurements (see details in Appendix A.2).

does obviously not take into account the impact of the magnetic activity, it is very surprising to observe such a weak excess. However, following our comment above, we believe that this is a consequence of reduction of $\mathcal{M}_{\mathrm{a}}$ by the magnetic activity, which led to values of $\mathcal{M}_{\mathrm{a}}$ for the active F-dwarfs comparable with what is expected for G-dwarfs stars. This would explain why $\sigma$ is mainly controlled by the term $T_{\mathrm{eff}}^{3 / 4} M^{-1 / 2} v_{\max }^{-1 / 2}$, from G-dwarfs to F-dwarfs, that is, the classical scaling relation.

The discrepancies observed for F-dwarfs stars must be explained by missing physical processes that involve the magnetic field at some level. To test the effect of a local magnetic field on the granulation background, Ludwig et al. (2009a) have computed a set of 2D MHD solar models with different magnetic flux levels. However, a negligible effect on the temporal power spectra of the emergent intensity was obtained, which leads the authors to conclude that locally generated magnetic fields are unlikely to be responsible for the discrepancy with the observations. We are then left with an enigmatic discrepancy.

\section{Summary and concluding remarks}

Using a grid of 3D models of the surface layers of various stars, we have computed the theoretical power density spectra associated with the granulation background on the basis of the theoretical model presented in Paper I. For each theoretical PDS we derived a characteristic time $\tau_{\text {eff }}$ and amplitude $\sigma$ associated with the granulation background. We compared these values with the theoretical scaling relations and observations.

From the current theoretical model we derived theoretical scaling relations for $\tau_{\text {eff }}$ and $\sigma: \tau_{\text {eff }}$ was found to scale inversely as $z_{2} \propto\left(\mathcal{M}_{\mathrm{a}} v_{\max }\right)$, while $\sigma$ was found to scale as $z_{3} \propto$ $\left(T_{\text {eff }}^{3 / 4} / M^{-1 / 2}\right) v_{\max }^{-1 / 2} f^{2}\left(\mathcal{M}_{\mathrm{a}}\right)$, where $\mathcal{M}_{\mathrm{a}}$ is the turbulent Mach number at the photosphere and $f\left(\mathcal{M}_{\mathrm{a}}\right)$ a second-order polynomial function (Eq. (12)). Both scaling relations were found to approximately agree with the individual values derived from the theoretical PDS obtained with our grid of 3D models.

The scaling relations predicted by the model depend on $v_{\max }$ in the same way as the classical theoretical scaling relations. Our model thus provides theoretical support for the scaling relation for $\tau_{\text {eff }}$ that was up to now explained by assuming that the granules move proportionally to the sound speed $c_{\mathrm{s}}$ (Huber et al. 2009; Kjeldsen \& Bedding 2011). Furthermore, consistently with the prediction by Ludwig (2006), our theoretical scaling relation for $\sigma$ is found to scale as the inverse of the square root of the number of granules over the stellar surface, which is expected to scale as $v_{\max } M / T_{\mathrm{eff}}^{3 / 2}$.

The model also predicts that $\tau_{\text {eff }}$ and $\sigma$ not only depend on $v_{\text {max }}$, but are also controlled by the Mach number $\mathcal{M}_{\mathrm{a}}$. For $\tau_{\text {eff }}$ this dependence on $\mathcal{M}_{\mathrm{a}}$ is simply explained by the fact that the granule life-time is ultimately controlled by the ratio between the granule size $\Lambda$ and its velocity $V$. In turn, this ratio is proportional to the inverse of the product $v_{\max } \mathcal{M}_{\mathrm{a}}$, where $\mathcal{M}_{\mathrm{a}} \propto V / c_{\mathrm{s}}$. For red giants, the observed dependence of $\tau_{\text {eff }}$ with $v_{\max }$ is thus explained by the fact that, during the evolution, variations of $v_{\max }$ dominate the variation of $\mathcal{M}_{\mathrm{a}}$. The dependence of $\sigma$ with $\mathcal{M}_{\mathrm{a}}$ is due to the close link between $\sigma$ and the temperature fluctuations $\theta_{\mathrm{rms}}$. In turn, there is a balance between $\theta_{\mathrm{rms}}$ and the granule kinetic energy (see Eq. (11)), and consequently between $\theta_{\text {rms }}$ and $\mathcal{M}_{\mathrm{a}}$.

To compare these theoretical scaling relations with the observations we have derived a scaling relation for $\mathcal{M}_{\mathrm{a}}$ using our grid of 3D models. This scaling has the form $\mathcal{M}_{\mathrm{a}} \propto T_{\text {eff }}^{2.4} g^{-0.15}$. We then compared the theoretical scaling relations for $\tau_{\text {eff }}$ and $\sigma$ with the measured values.

Quantitatively, the theoretical values $\tau_{\text {eff }}$ systematically underestimate the measurements by about $40 \%$. This systematic difference is mainly a consequence of the way $\tau_{\text {eff }}$ is determined from the granulation spectrum (see Sect. 6.1). Calibrating the theoretical $\tau_{\text {eff }}$ to the solar reference results in a difference with the observations of less than $10 \%$ on average. At fixed values of $z_{3}$, theoretical values of $\sigma$ systematically overestimate the observations made for red giants by about $12 \%$. For a large part, this overestimation was shown to be the consequence of the considerable degeneracy that occurs for red giants between the mass and radius (see Appendix B). Comparing instead the quantity $\tilde{\sigma} \equiv\left(R_{\mathrm{S}} / R_{\odot}\right) \sigma$ removes the dependence with the masses and radii attributed to the $3 \mathrm{D}$ models and results on average in a difference with the measurements of only 5\%. For MS and subgiants, the theoretical $\sigma$ differ on average by about $50 \%$ from the measurements. This departure is found to depend on $T_{\text {eff }}$. The highest deviations are obtained with the F-dwarf stars and are very likely due to the fact that the impact of the magnetic activity on the granulation background is not modelled (see the discussion in Sect. 7). If we exclude the F-dwarf stars from our sample, theoretical $\sigma$ underestimate the observations by only about $2 \%$.

For RG stars, the differences between our predictions for $\sigma$ and $\tau_{\text {eff }}$ and the measurements are found to be lower than the differences obtained with the different methods of analysis investigated in Mathur et al. (2011). For $\sigma$, these differences remain much lower than those obtained by Mathur et al. (2011). Indeed, theoretical calculations of these authors overestimate the measured $\sigma$ by a factor of about four. In view of our results, the Mathur et al. (2011) results are surprising since their theoretical calculations are based on the ab initio modelling of Ludwig (2006) and, as shown in Paper I, our theoretical 1D modelling agrees well with the theoretical PDS computed on the basis of the $\mathrm{ab}$ initio modelling for a red giant 3D model (Ludwig \& Steffen 2012).

The theoretical scaling relations derived from our model match the variations of $\tau_{\text {eff }}$ and $\sigma$ measured across the HR diagram with Kepler data on a global scale. Nevertheless, the differences between the new scaling relations and the classical ones are found to be of the same order as the dispersions of the new scaling relations w.r.t. the measurements. It is therefore not 
possible with our sample of stars to confirm or refute the dependence of $\sigma$ and $\tau_{\text {eff }}$ with $\mathcal{M}_{\mathrm{a}}$. The high levels of the dispersions between the scaling relations and the measurements have two origins. First, $\mathcal{M}_{\mathrm{a}}$ mainly depends on $T_{\text {eff }}$, and the precision with which $T_{\text {eff }}$ is measured significantly contributes to the dispersion. Second, we observed population of stars inhomogeneous in terms of surface metal abundance. However, the granulation background is expected to depend on the surface metal abundance in a manner that remains to be investigated across the HR diagram (for a particular low-metal F-dwarf star see Ludwig et al. 2009a).

Detection of solar-like oscillations in a statistically sufficient number of K-dwarf stars $\left(T_{\text {eff }}=3500-5000\right)$ would in principle permit us to test the dependence of $\tau_{\text {eff }}$ and $\sigma$ on $\mathcal{M}_{\mathrm{a}}$. Indeed, these cool dwarf stars have $v_{\max } \gtrsim 800 \mu \mathrm{Hz}(\log g \gtrsim 4.0)$ and are expected to have simultaneously a significantly lower $\mathcal{M}_{\mathrm{a}}$ and a $v_{\max }$ comparable to the MS stars for which solar-like oscillations were so far detected with Kepler. Unfortunately, such K-dwarf stars are lacking in our sample.

In Chaplin et al. (2011b), about 760 stars showing solarlike oscillations and observed with a short cadence were flagged by the detection threshold. Of these targets, those with $v_{\max } \gtrsim$ $0.8 \mathrm{mHz}$ all have an effective temperature higher than $5000 \mathrm{~K}$. It is therefore very unlikely to extend the current samples such as to have a statistically sufficient number of K-dwarf stars showing solar-like oscillations. If we can verify the dependence of the scaling relations on $\mathcal{M}_{\mathrm{a}}$, this will constitute a confirmation of the theoretical scaling relations derived in the present work. It will also constitute a way to test for a variety of stellar standard 1D models of the surface convection.

Acknowledgements. Funding for this Discovery mission is provided by NASA's Science Mission Directorate. The authors thank the entire Kepler team, without whom these results would not be possible. R.S., K.B., B.M. and A.B. acknowledge financial support from the Programme National de Physique Stellaire (PNPS) of CNRS/INSU and from Agence Nationale de la Recherche (ANR, France) program "Interaction Des Étoiles et des Exoplanètes" (IDEE, ANR-12-BS05-0008). E.C. and H.G.L. acknowledge financial support by the Sonderforschungsbereich SFB 881 "The Milky Way System" (subproject A4) of the German Research Foundation (DFG). The National Center for Atmospheric Research (NCAR) is partially funded by the National Science Foundation. SM's work was partially supported by NASA grant NNX12AE17G.

\section{References}

Aigrain, S., Favata, F., \& Gilmore, G. 2004, in Stellar Structure and Habitable Planet Finding, eds. F. Favata, S. Aigrain, \& A. Wilson, ESA SP, 538, 215

Andersen, B., Leifsen, T., Appourchaux, T., et al. 1998, in Structure and Dynamics of the Interior of the Sun and Sun-like Stars, ed. S. Korzennik, ESA SP, 418, 83

Asplund, M., Grevesse, N., \& Sauval, A. J. 2005, in Cosmic Abundances as Records of Stellar Evolution and Nucleosynthesis, eds. T. G. Barnes, III, \& F. N. Bash, ASP Conf. Ser., 336, 25

Ballot, J., Barban, C., \& van't Veer-Menneret, C. 2011, A\&A, 531, A124 Bedding, T. R., \& Kjeldsen, H. 2003, PASA, 20, 203

Belkacem, K., Goupil, M. J., Dupret, M. A., et al. 2011, A\&A, 530, A142

Belkacem, K., Samadi, R., Mosser, B., Goupil, M.-J., \& Ludwig, H.-G. 2013, in

Progress in physics of the sun and stars: a new era in helio- and asteroseismology, ASP Conf. Ser. [arXiv: 1307.3132]

Brown, T. M., Gilliland, R. L., Noyes, R. W., \& Ramsey, L. W. 1991, ApJ, 368, 599

Brown, T. M., Latham, D. W., Everett, M. E., \& Esquerdo, G. A. 2011, AJ, 142, 112
Bruntt, H., Frandsen, S., \& Thygesen, A. O. 2011, A\&A, 528, A121 Bruntt, H., Basu, S., Smalley, B., et al. 2012, MNRAS, 423, 122 Chaplin, W. J., Bedding, T. R., Bonanno, A., et al. 2011a, ApJ, 732, L5 Chaplin, W. J., Kjeldsen, H., Bedding, T. R., et al. 2011b, ApJ, 732, 54 Cox, J. 1968, Principles of stellar structure (Gordon and Breach)

Freytag, B., Steffen, M., Ludwig, H.-G., et al. 2012, J. Comput. Phys., 231, 919

García, R. A., Mathur, S., Salabert, D., et al. 2010, Science, 329, 1032

García, R. A., Hekker, S., Stello, D., et al. 2011, MNRAS, 414, L6

Guenther, D. B., Kallinger, T., Gruberbauer, M., et al. 2008, ApJ, 687, 1448

Hansen, C. J., \& Kawaler, S. D. 1994, Stellar Interiors. Physical Principles, Structure, and Evolution

Harvey, J. 1985, in Future Missions in Solar, Heliospheric \& Space Plasma Physics, eds. E. Rolfe, \& B. Battrick, ESA SP, 235, 199

Harvey, J. W., Duvall, Jr., T. L., Jefferies, S. M., \& Pomerantz, M. A. 1993, in GONG 1992. Seismic Investigation of the Sun and Stars, ed. T. M. Brown, ASP Conf. Ser., 42, 111

Hekker, S., Elsworth, Y., De Ridder, J., et al. 2011, A\&A, 525, A131

Houdek, G., Balmforth, N. J., Christensen-Dalsgaard, J., \& Gough, D. O. 1999, A\&A, 351, 582

Huber, D., Stello, D., Bedding, T. R., et al. 2009, Commun. Asteroseismol., 160, 74

Kallinger, T., \& Matthews, J. M. 2010, ApJ, 711, L35

Kallinger, T., Weiss, W. W., Barban, C., et al. 2010, A\&A, 509, A77

Karoff, C. 2012, MNRAS, 421, 3170

Karoff, C., Campante, T. L., Ballot, J., et al. 2013, ApJ, 767, 34

Kjeldsen, H., \& Bedding, T. R. 1995, A\&A, 293, 87

Kjeldsen, H., \& Bedding, T. R. 2011, A\&A, 529, L8

Ludwig, H. 2006, A\&A, 445, 661

Ludwig, H.-G., \& Steffen, M. 2012, 3D Model Atmospheres of Red Giant Stars, Red Giants as Probes of the Structure and Evolution of the Milky Way, eds. A. Miglio, J. Montalbán, \& A. Noels (Berlin, Heidelberg: Springer-Verlag), Astrophys. Space Sci. Proc., 125

Ludwig, H., Samadi, R., Steffen, M., et al. 2009a, A\&A, 506, 167

Ludwig, H.-G., Caffau, E., Steffen, M., et al. 2009b, Mem. Soc. Astron. It., 80, 711

Mathur, S., Hekker, S., Trampedach, R., et al. 2011, ApJ, 741, 119

Michel, E., Baglin, A., Auvergne, M., et al. 2008, Science, 322, 558

Michel, E., Samadi, R., Baudin, F., et al. 2009, A\&A, 495, 979

Molenda-Żakowicz, J., Bruntt, H., Sousa, S., et al. 2010, Astron. Nachr., 331, 981

Morel, P., \& Lebreton, Y. 2008, Ap\&SS, 316, 61

Morel, T., \& Miglio, A. 2012, MNRAS, 419, L34

Mosser, B., Bouchy, F., Catala, C., et al. 2005, A\&A, 431, L13

Mosser, B., Baudin, F., Lanza, A. F., et al. 2009, A\&A, 506, 245

Mosser, B., Belkacem, K., Goupil, M.-J., et al. 2010, A\&A, 517, A22

Mosser, B., Elsworth, Y., Hekker, S., et al. 2012, A\&A, 537, A30

Mosser, B., Michel, E., Belkacem, K., et al. 2013, A\&A, 550, A126

Nordlund, A., Stein, R. F., \& Asplund, M. 2009, Liv. Rev. Sol. Phys., 6, 2

Noyes, R. W., Hartmann, L. W., Baliunas, S. L., Duncan, D. K., \& Vaughan, A. H. 1984, ApJ, 279, 763

Pinsonneault, M. H., An, D., Molenda-Żakowicz, J., et al. 2012, ApJS, 199 , 30

Samadi, R., Ludwig, H.-G., Belkacem, K., et al. 2010a, A\&A, 509, A16

Samadi, R., Ludwig, H.-G., Belkacem, K., Goupil, M. J., \& Dupret, M.-A. 2010b, A\&A, 509, A15

Samadi, R., Belkacem, K., \& Ludwig, H.-G. 2013, A\&A, 559, A39

Silva Aguirre, V., Casagrande, L., Basu, S., et al. 2012, ApJ, 757, 99

Stello, D., Chaplin, W. J., Basu, S., Elsworth, Y., \& Bedding, T. R. 2009, MNRAS, 400, L80

Svensson, F., \& Ludwig, H.-G. 2005, in 13th Cambridge Workshop on Cool Stars, Stellar Systems and the Sun, eds. F. Favata, G. A. J. Hussain, \& B. Battrick, ESA SP, 560, 979

Thygesen, A. O., Frandsen, S., Bruntt, H., et al. 2012, A\&A, 543, A160

Trampedach, R. 2004, Ph.D. Thesis, Michigan State University

Trampedach, R., Christensen-Dalsgaard, J., Nordlund, A., \& Stein, R. F. 1998, in The First MONS Workshop: Science with a Small Space Telescope, eds. H. Kjeldsen, \& T. R. Bedding, 59

Tremblay, P.-E., Ludwig, H.-G., Freytag, B., Steffen, M., \& Caffau, E. 2013, A\&A, 557, A7

Vázquez Ramió, H., Régulo, C., \& Roca Cortés, T. 2005, A\&A, 443, L11

Pages 11 to 12 are available in the electronic edition of the journal at http://www . aanda.org 


\section{Appendix A: Can we distinguish the dependence on $\mathcal{M}_{\mathrm{a}}$ ?}

We have seen that the new theoretical scaling relations for $\tau_{\text {eff }}$ and $\sigma$ are - on a global scale - aligned with the Kepler measurements. However, the question we address here is whether or not the observations allow one to quantitatively confirm the dependence of the new scaling relations on $\mathcal{M}_{\mathrm{a}}$. To this end we compare the new scaling relations with the classical ones.

\section{A.1. Characteristic time-scale, $\tau_{\text {eff }}$}

To check the dependence of the new theoretical scaling relation for $\tau_{\text {eff }}$ on $\mathcal{M}_{\mathrm{a}}$, we computed the relative differences between the new scaling relation and the measurements as well as the relative differences between the classical theoretical scaling relation $\tau_{\text {eff }} \propto v_{\max }^{-1}$ and the measurements. In practice, we computed the quantities $D_{\tau}=\left(\tau_{\text {eff }, \odot} / \tau_{\text {eff,m }}\right) z_{2}-1$ and $D_{\tau}^{\prime}=\left(\tau_{\text {eff } \odot} / \tau_{\text {eff,m }}\right) c_{2}-1$, where $\tau_{\text {eff,m }}$ is the measured value of $\tau_{\text {eff }}, \tau_{\text {eff, } \odot}=230 \mathrm{~s}$ is the adopted solar reference (Michel et al. 2008), $c_{2}=\left(v_{\text {ref }} / v_{\max }\right)$, and $z_{2}$ is the new scaling relation (Eq. (18)). We considered in our comparison only the sample of MS and sub-giant stars because they are better indicator for the dependence on $\mathcal{M}_{\mathrm{a}}$.

The histograms of $D_{\tau}$ and $D_{\tau}^{\prime}$ are shown in Fig. A.1 (top panel). The median value and the standard deviation of $D_{\tau}$ are $-10 \%$ and $12 \%$, respectively, while for $D_{\tau}^{\prime}$ they are equal to $15 \%$ and $14 \%$, respectively.

For both scaling relations, the dispersion and deviation from the measurements can in part arise because we observed an heterogeneous population of stars, in particular stars with different metal abundance. Indeed, $\mathcal{M}_{\mathrm{a}}$ is expected to depend on the surface metal abundance (see e.g. Houdek et al. 1999; Samadi et al. 2010b,a). However, we would have expected a higher dispersion for $D_{\tau}$ than for $D_{\tau}^{\prime}$. Indeed, the new scaling relation depends on $\mathcal{M}_{\mathrm{a}}$ and, according to Eq. (17), the Mach number $\mathcal{M}_{\mathrm{a}}$ strongly depends on $T_{\text {eff }}$ and more weakly on $g$. Therefore, the uncertainties associated with $T_{\text {eff }}$ and $\log g$ introduce a spread in the determination of $z_{2}$, and subsequently on $D_{\tau}$.

$T_{\text {eff }}$ is based on photometric indices and is measured with an rms precision of about $100 \mathrm{~K}$ (see Molenda-Żakowicz et al. 2010; Bruntt et al. 2011, 2012; Thygesen et al. 2012), while $\log g$ is obtained from seismology with a typical rms precision of 0.1 dex (Bruntt et al. 2012; Morel \& Miglio 2012). The rms errors in $T_{\text {eff }}$ and $\log g$ introduce a relative dispersion in $z_{2}$ of the order of $6 \%$ for a typical RG star with $T_{\text {eff }}=4500 \mathrm{~K}$ and $\log g=2.3$, and about $5 \%$ for a typical MS with $T_{\text {eff }}=6000 \mathrm{~K}$ and $\log g=4$ (these typical relative dispersions are shown in Fig. 4).

The median deviation of the new theoretical scaling relation from the measurements is found to be of the same order as that of the classical relation. However, the difference between the median of $D_{\tau}$ and of $D_{\tau}^{\prime}$ remains within the dispersion of $D_{\tau}$. Therefore, we cannot distinguish the new scaling relation from the classical one. Finally, the mean deviation of the new scaling relation from the measurements is about two times lower than its associated dispersion. We therefore conclude that, as the classical scaling relation, the new one is compatible with the observations, but we cannot firmly confirm its dependence with $\mathcal{M}_{\mathrm{a}}$.

\section{A.2. Brightness fluctuations, $\sigma$}

In the same way as for $\tau_{\text {eff }}$, we checked the dependence on $\mathcal{M}_{\mathrm{a}}$ of the new scaling relation for $\sigma$ by computing the relative difference between the new theoretical scaling relation (Eq. (19)) and
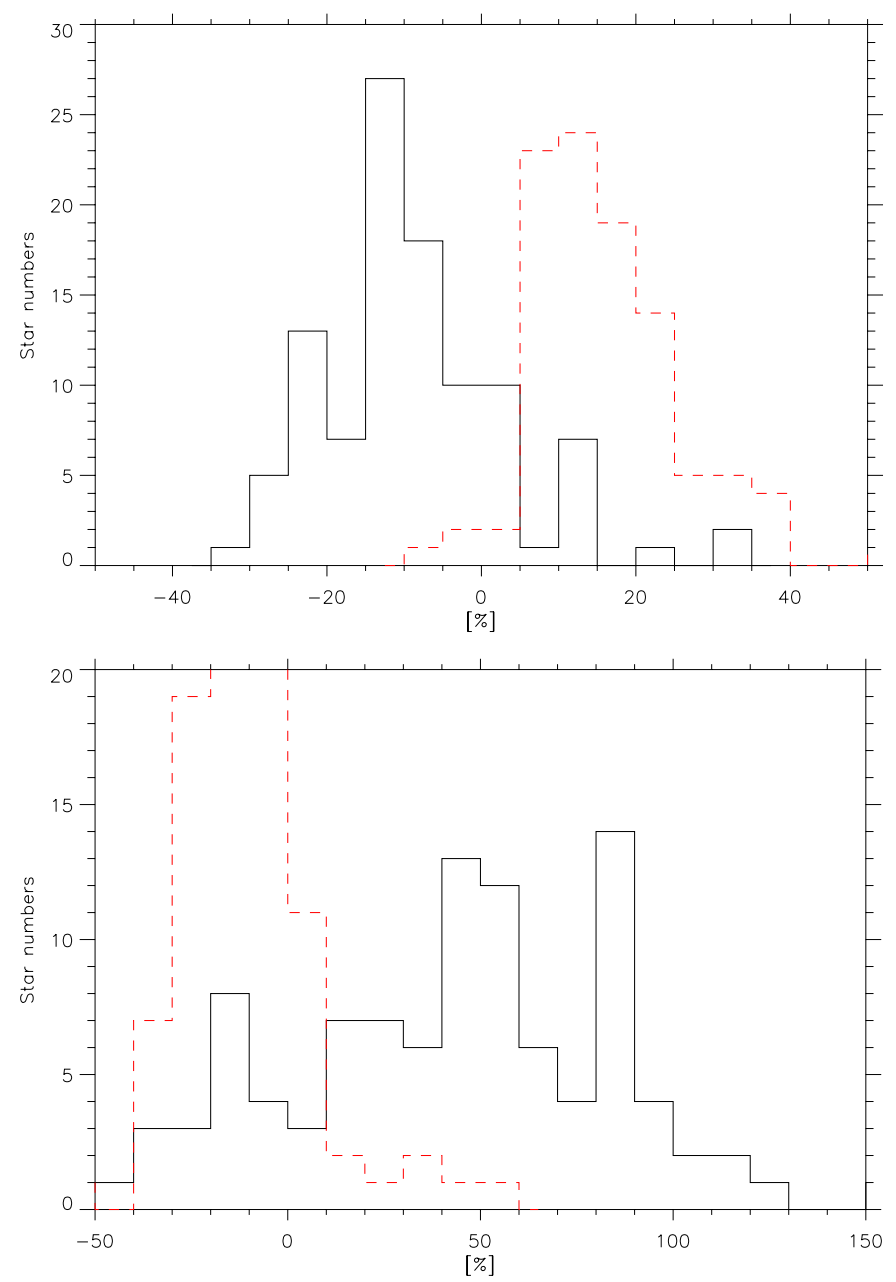

Fig. A.1. Top: histogram of the relative differences (in \%) between the theoretical $\tau_{\text {eff }}$ and the measured ones. The solid black line corresponds to the histogram of the relative difference $D_{\tau}=\left(\tau_{\text {eff, } \odot} / \tau_{\text {eff, }}\right) z_{2}-1$ and the dashed red line to residuals $D_{\tau}^{\prime}=\left(\tau_{\text {eff, } \odot} / \tau_{\text {eff, }}\right) c_{2}-1$, where $z_{2}=\left(v_{\text {ref }} / v_{\max }\right)\left(\mathcal{M}_{\mathrm{a}, 0} / \mathcal{M}_{\mathrm{a}}\right)$ is our theoretical scaling relation and $c_{2}=$ $\left(v_{\text {ref }} / v_{\max }\right)$ the classical one. Bottom: histogram of the relative differences (in \%) between the theoretical $\sigma$ and the measured ones. The solid black line correspond to the histogram of the relative difference $D_{\sigma}=\left(\sigma_{\odot} / \sigma_{\mathrm{m}}\right) z_{3}-1$ and the dashed red line to the relative difference $D_{\sigma}^{\prime}=\left(\sigma_{\odot} / \sigma_{\mathrm{m}}\right) c_{3}-1$, where $z_{3}$ (Eq. (19)) is the new theoretical scaling relation and $c_{3}$ (Eq. (20)) is the classical one.

the measurements as well as the relative difference between the classical theoretical scaling relation $T_{\mathrm{eff}}^{3 / 4} M^{-1 / 2} v_{\max }^{-1 / 2}$ (Kjeldsen \& Bedding 2011; Mathur et al. 2011) and the measurements. In practice, we computed the quantities $D_{\sigma}=\left(\sigma_{\odot} / \sigma_{\mathrm{m}}\right) z_{3}-1$ and $D_{\sigma}^{\prime}=\left(\sigma_{\odot} / \sigma_{\mathrm{m}}\right) c_{3}-1$, where $\sigma_{\mathrm{m}}$ is the measured value of $c_{2} \propto \sigma, \sigma_{\odot}=43 \mathrm{ppm}$ is the adopted bolometric amplitude measured for the Sun (Michel et al. 2008), and $c_{3}$ is given by Eq. (20), where the term $\left(T_{\text {eff }} / T_{\text {eff, } \odot}\right)^{3 / 4}\left(M_{\odot} / M\right)^{1 / 2}$ is evaluated according to Eq. (22). Like for $\tau_{\text {eff }}$, we considered only MS and sub-giant stars because they are the better indicators.

We have plotted in Fig. A.1 (bottom panel) the histograms associated with $D_{\sigma}$ and $D_{\sigma}^{\prime}$. The median value and standard deviation of $D_{\sigma}$ are $46 \%$ and $42 \%$, respectively, while for $D_{\sigma}^{\prime}$ they are equal to $-12 \%$ and $17 \%$, respectively.

As mentioned for the scaling of $\tau_{\text {eff }}$ (see Sect. 6.1), for both scaling relations, the dispersion and deviation with the measurements can in part arise from the fact that we observed an inhomogeneous sample of stars, in particular, stars with a different 
surface metal abundances. Indeed, the amplitude of the granulation background is expected to depend on the surface metal abundance (for a particular low-metal F-type star see Ludwig et al. 2009a). Furthermore, for the new scaling relation an rms error of $100 \mathrm{~K}$ in $T_{\text {eff }}$ and a rms error 0.1 dex on $\log g$ results for $z_{3}$ in a typical error about $12 \%$ for RG stars and about $10 \%$ for a typical MS (these typical relative dispersions are shown in Fig. 5). On the other hand, the uncertainties associated with $T_{\text {eff }}$ have no direct impact on the classical scaling relation given by Eq. (20) since the term $T_{\text {eff }}^{3 / 2} M^{-1 / 2}$ is estimated using only seismic constraints (see Eq. (22)).

Compared with the new scaling relation, the classical one results in a smaller difference with the observations. However, the deviations of the two scaling relations from the measurements are found to depend on $T_{\text {eff }}$. The highest deviations are obtained for the F-dwarf stars $\left(T_{\text {eff }}=6000-7500 \mathrm{~K}\right.$, see Fig. 6 and Sect. 7). As discussed in Sect. 7, this is very likely a consequence of the lack of modelling of the impact of magnetic activity on the granulation background.

As stressed in Paper I, our theoretical calculations are expected to be valid for stars with a low level of activity. If we exclude the F-dwarf stars from our sample, the median deviation of the new scaling relation w.r.t the measurements is $-2 \%$ $( \pm 30 \%)$, while for the classical scaling relation it is equal to $-19 \%( \pm 18 \%)$. In that case, the new scaling relation results in a lower deviation. However, the difference between the median value of $D_{\sigma}$ and this of $D_{\sigma}^{\prime}$ is smaller than the standard deviation of $D_{\sigma}$. Therefore, it is not possible to distinguish the new theoretical scaling relation from the classical one.

In conclusion, as the classical scaling relation, our theoretical scaling relation is compatible with the observations, but we cannot confirm the dependence on $\mathcal{M}_{\mathrm{a}}$. Observations of $\mathrm{K}$ dwarf stars $\left(T_{\text {eff }}=3500-5000 \mathrm{~K}\right)$ could in principle help to check the dependence of the theoretical scaling relation on $\mathcal{M}_{\mathrm{a}}$. Indeed, for instance the 3D model with $T_{\text {eff }} \simeq 4500 \mathrm{~K}$ and $\log g=4.0$ (K dwarf) has $v_{\max }=1.3 \mathrm{mHz} \mathcal{M}_{\mathrm{a}} \simeq 0.18$, and $\sigma \simeq 18$ ppm, while the $3 \mathrm{D}$ model $T_{\text {eff }} \simeq 5900 \mathrm{~K}$ and same log $g$ (G dwarf) has $v_{\max }=1.1 \mathrm{mHz} \mathcal{M}_{\mathrm{a}} \simeq 0.31$, and $\sigma \simeq 110 \mathrm{ppm}$. The relative difference in $\sigma$ between the $\mathrm{K}$ dwarf model and the G-dwarf model is $84 \%$. This is much higher than the dispersion in $D_{\sigma}$ and $D_{\sigma}^{\prime}$.

\section{Appendix B: Removing the degeneracy with the mass and the radius}

As seen in Sect. 4.4, the individual theoretical values of $\sigma$ are found to scale as $z_{3}^{p}$ with the slope $p=1.10$. As we will show now, the deviation of the individual values of $\sigma$ from a linear scaling with $z_{3}$ is for a large part due to the considerable degeneracy that occurs for red giants between $M$ and $R$. Indeed, the theoretical values of $\sigma$ scale as $\mathcal{N}_{\mathrm{g}}^{-1 / 2}$, and hence as the stellar radius $R_{\mathrm{S}}$ (see Eqs. (4) and (7)). Furthermore, $z_{3}$ scales as $M^{-1 / 2}$. Therefore theoretical values of $\sigma$ and $z_{3}$ directly depend on the masses and radii attributed to the $3 \mathrm{D}$ models. However, two red giants with same $T_{\text {eff }}$ and $\log g$ can have very different values

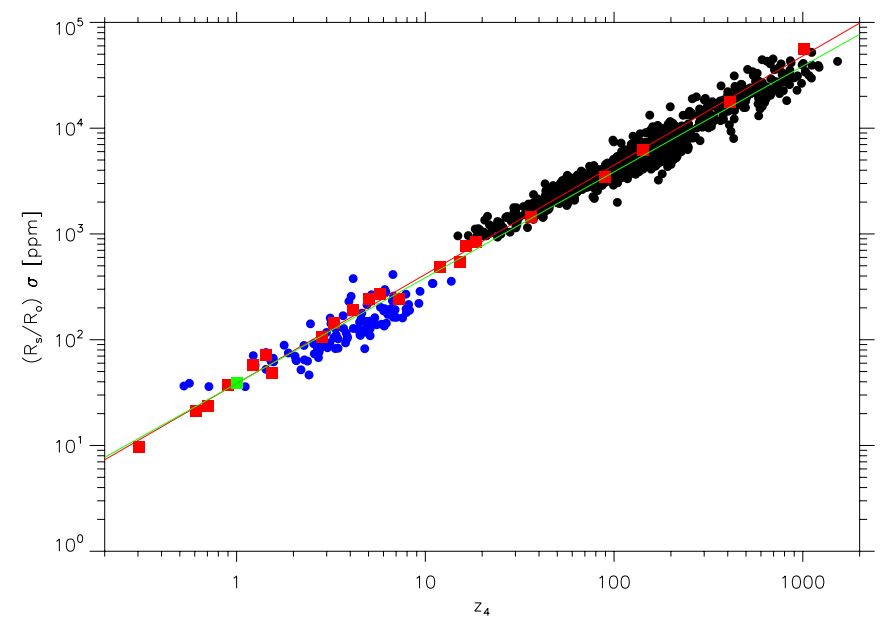

Fig. B.1. $\tilde{\sigma} \equiv\left(R_{\mathrm{S}} / R_{\odot}\right) \sigma$ as a function the quantity $z_{4}$ given by Eq. (B.2). The symbols have the same meaning as in Fig. 1. The green line corresponds to a linear scaling with $z_{4}$ and the red one to a power law of the form $z_{4}^{p}$ where the slope $p=1.03$ is obtained by fitting the individual theoretical values of $\tilde{\sigma}$ (red squares).

of $R$ and $M$. Furthermore, the masses and radii attributed to our 3D models were obtained from a grid of standard stellar models with fixed physical assumptions, and all of these models are in the pre-helium-burning phase, which is not the case for all observed RG stars.

When we multiply theoretical $\sigma$ by $R_{\mathrm{S}} / R_{\odot}$, we obtain a quantity that does no longer depend on the radius attributed to the $3 \mathrm{D}$ model. Furthermore, the quantity $z_{4} \equiv z_{3}\left(R_{\mathrm{S}} / R_{\odot}\right)$ scales as $g^{-1 / 2}$. As a consequence, $z_{4}$ does not depend on the mass attributed to the 3D model. To remove possible bias introduced by the determination of the masses and radii of the 3D models we must therefore compare theoretical values of $\tilde{\sigma}$ as a function of $z_{4}$ with the measurements multiplied by the star radii. To do this, we need to determine the radii of the observed targets. Combining the scaling relation for $v_{\max }$ with the one for $\Delta v$ gives (see e.g. Stello et al. 2009; Kallinger et al. 2010; Mosser et al. 2010)

$$
\frac{R_{\mathrm{S}}}{R_{\odot}}=\left(\frac{v_{\text {max }}}{v_{\text {ref }}}\right)\left(\frac{\Delta v}{\Delta v_{\text {ref }}}\right)^{-2}\left(\frac{T_{\text {eff }}}{T_{\text {eff }, \odot}}\right)^{1 / 2} .
$$

Multiplying Eq. (19) by Eq. (B.1) gives the scaling relation for $\tilde{\sigma}=\left(R_{\mathrm{S}} / R_{\odot}\right) \sigma$ with the help of Eq. (22)

$\tilde{\sigma} \propto z_{4}=\left(\frac{v_{\text {ref }}}{v_{\max }}\right)\left(\frac{f\left(\mathcal{M}_{\mathrm{a}}\right)}{f\left(\mathcal{M}_{\mathrm{a}, 0}\right)}\right)^{2}$.

To compare theoretical $\tilde{\sigma}$ with the measurements, we multiply the measured $\sigma$ by the ratio $R_{\mathrm{S}} / R_{\odot}$ given by Eq. (B.1). We have plotted theoretical and measured values of $\tilde{\sigma}$ in Fig. B.1. The individual theoretical values of $\tilde{\sigma}$ are found to scale as $z_{4}^{p}$ with $p=1.03$ and are therefore better aligned with the measurements than those of $\sigma$. 